\title{
Generalized Modifications and Cobounding Manifolds
}

\author{
M. V. MIELKE
}

\author{
Communicated by A. H. WALLACE
}

0. Introduction. In [12] and [13] Wallace studies the relationship between cobounding manifolds and spherical modifications. In $\$ 1$ we study a more general kind of modification; namely, the (closed) symmetric difference of two manifolds. If $M_{1}$ and $N$ are two intersecting $n$-manifolds such that the symmetric difference is again an $n$-manifold $M_{2}$ we denote $M_{2}$ by $M_{1} \Delta N$ and say that $M_{2}$ is obtained by modifying $M_{1}$ by $N$. In $\S 2$, we obtain relationships among the homotopy groups of $M_{1}, N, M_{2}$.

In $\S 4$ we specialize to the case that $N$ is an $n$-sphere $S^{n}$. If certain restrictions are put on $M_{1} \cap S^{n}$ then $M_{1} \Delta S^{n}$ is a spherical modification as defined in [12, 504]. The general results on modifications then yield the important theorem on homotopy groups of $M_{1}$ and $M_{2}$ (Theorem 11.3). In $\$ 13$ we give conditions under which a generalized modification can be realized by a finite sequence of spherical modifications and in $\$ 14$ some results are obtained on the existance of manifolds with modification number 1 ; that is, mainfolds upon which functions exist having exactly three critical points.

In [13, 800-801] Wallace introduces the notions of a basic complex and a semimodification and uses them to derive certain results on the kinds of spherical modifications needed to go from one manifold to another if both manifolds and the manifold they bound contain a basic complex. (Cf. [13] Theorem 10.1) An essential ingredient in the proof of Theorems 9.6, 9.7 and 10.3 of [13] is a certain characteristic map $\Phi$ defined by Wallace in $[13,786]$. In $\$ 9$ we define another map (Case 1) and exhibit its relationship to the map $\Phi$ (Case 2). This new map enables us to replace the notion of a basic complex by the notion of standard homology form. Theorem 15.1 gives us that any manifold cobounds a manifold in standard homology form and the proof of the theorem gives an algorithm for putting a given manifold in such a form. The corresponding theorem for basic complexes is proven in [13] in a sequence of steps (Lemma 9.1 through 9.5 and Theorems 9.6 and 9.7 ) by means of semimodifications. The elimination of the use of semimodifications in our method is essentially due to the presence of Theorem 11.3. Theorem 15.2 gives the theorem corre- 
sponding to Theorem 10.1 [13] and a further analysis of Case 1 and Case 2. $\$ 9$ allows us to compute the homology groups of a basic complex in Theorem 15.3.

I would like to thank A. H. Wallace for his assistance in the preparation of this paper.

1. Modifications of Manifolds. Although the following results are true for more general kinds of topological spaces, we will only be concerned with connected differentiable manifolds to be referred to simply as manifolds.

Let $M_{1}, N$ be $n$-dimensional manifolds. Define $M_{2}=$ closure

$$
\left\{\left(M_{1}-M_{1} \cap N\right) \cup\left(N-M_{1} \cap N\right)\right\} .
$$

$M_{2}$ is the closure of the usual symmetric difference of two sets, closure being necessary to insure connectivity of $M_{2}$ assuming $M_{1} \cap N \neq 0$. If $M_{1}$ and $N$ intersect in such a way that $M_{2}$ is again an $n$-manifold then $M_{2}$ will be called the manifold obtained by performing a modification of $M_{1}$ by $N$ and we shall write $M_{2}=M_{1} \Delta N$. To compute $M_{2}$ it is not only necessary to describe $M_{1}$ and $N$ but also how they intersect. This will be made more explicit in $\$ 4$.

2. Homotopy Groups. We now set about to determine the relationship among the homotopy groups of $M_{1}, N$ and $M_{2}$. Suppose $M_{1}$ and $N$ are given such that $M_{1} \Delta N$ exists. Define $X=M_{1} \cup N, B_{1}=M_{1} \cap N, B_{2}=$ closure $\left(N-B_{1}\right)$. Hence $M_{2}=\left(M_{1}-B_{1}\right) \cup B_{2}$ and the relationship of these sets to each other is shown in the following commutative diagram where all arrows are inclusions.

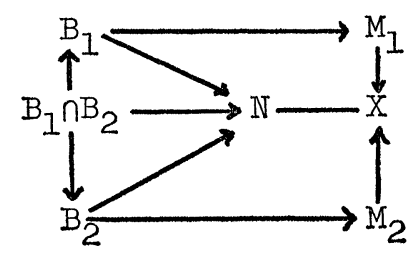

Since $N$ is connected, $B_{1} \cap B_{2} \neq 0$. We pick a point $x$ in $B_{1} \cap B_{2}$ as the base point for the homotopy groups. It is obvious from the above diagram that $x$ is in all the sets concerned. Having once picked the point $x$ it will no longer be mentioned.

Let $\Gamma_{i}$ be the largest integer such that for all integers $k<\Gamma_{i}, \Pi_{k}\left(B_{i}\right)=0$ for $i=1,2$. Let $\Gamma_{i+2}$ be the largest integer such that for all integers $k<\Gamma_{i+2}$ the map $i: \Pi_{k}\left(B_{i}\right) \rightarrow \Pi_{k}(N)$ induced by inclusion is an epimorphism for ${ }_{i}=1,2$. Let $\Gamma_{5}$ be the largest integer such that for all integers $k<\Gamma_{5}, \Pi_{k}(N)=0$. Consider the following exact sequence of groups where those on both ends are the triad groups of Massey [2]:

$\rightarrow \Pi_{k+1}\left(X ; M_{i}, N\right) \stackrel{\delta_{k+1, i}}{\rightarrow} \Pi_{k}\left(M_{i}, B_{i}\right) \stackrel{\gamma_{k, i}}{\rightarrow} \Pi_{k}(X, N) \stackrel{j_{k, i}}{\rightarrow} \Pi_{k}\left(X ; M_{i} N\right) \rightarrow, \quad i=1,2$.

Sequence I

Lemma 2.1. $\Pi_{k}\left(X ; M_{i}, N\right)=0$ if $2 \leqq k<\min \left(\Gamma_{i}+1, \Gamma_{i+2}\right), i=1,2$. 
Proof. By [2] one has that the obstruction to making $\Pi_{k}\left(X ; M_{i}, N\right)$ equal zero lies in a cohomology group with coefficients in $\Pi_{k}\left(N, B_{i}\right), i=1.2$. Consider the following exact sequence

$$
\rightarrow \Pi_{k}\left(B_{i}\right) \rightarrow \Pi_{k}(N) \rightarrow \Pi_{k}\left(N, B_{i}\right) \rightarrow \Pi_{k-1}\left(B_{i}\right) \rightarrow
$$

Thus $\Pi_{k}\left(N, B_{i}\right)=0$ if $k<\Gamma_{i+2}$ and $k<\Gamma_{i}+1, i=1,2$, and the lemma follows.

Lemma 2.2. $\delta_{k, i}=0$ if $2 \leqq k<\Gamma_{i}+1, i=1,2$.

Proof. The obstruction to making $\delta_{k, i}$ equal zero lies in a cohomology group with coefficients in $\Pi_{k-1}\left(B_{i}\right)$. Thus, if $k<\Gamma_{i}+1$, then $\Pi_{k-1}\left(B_{i}\right)=0, i=1,2$, and the lemma follows.

Lemma 2.3. $\gamma_{k, i}$ is an isomorphism if $2 \leqq k<\min \left(\Gamma_{i}, \Gamma_{i+2}\right), i=1,2$.

Proof. The lemma follows from Lemma 2.1, Lemma 2.2 and the exactness of Sequence $I$.

It should be noted that for $k$ to exist we must have $\Gamma_{i}>2$ and $\Gamma_{i+2}>2$.

Theorem 2.1. Let $i: M_{2} \rightarrow X$ be the inclusion. Then $i_{*}: \Pi_{r}\left(M_{2}\right) \rightarrow \Pi_{r}(X)$ is an isomorphism for $2 \leqq r<\min \left(\Gamma_{2}, \Gamma_{4}, \Gamma_{5}\right)$. For $2 \leqq r<\Gamma_{2}$ the map $\gamma_{r, 2} j_{r}: \Pi_{r}\left(M_{2}\right) \rightarrow \Pi_{r}(X, N)$ is a monomorphism.

Proof. Consider the following commutative diagram where the horizontal sequences are exact and the vertical arrows are induced by inclusion of $\left(M_{2}, B_{2}\right)$ in $(X, N)$.

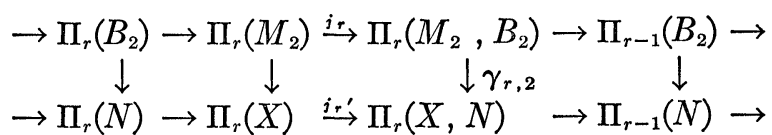

Diagram 1.

Then $j_{r}$ is an isomorphism if $r<\Gamma_{2}, j_{r}^{\prime}$ is an isomorphism if $r<\Gamma_{5}$ and $\gamma_{r, 2}$ is an isomorphism if $2 \leqq r<\min \left(\Gamma_{2}, \Gamma_{4}\right)$ by Lemma 2.3. This proves the first part of the theorem. By Lemma 2.2 and the exactness of Sequence I it follows that $\gamma_{r, 2}$ is a monomorphism if $2 \leqq r<\Gamma_{2}$. This gives the second part of the theorem.

Theorem 2.2. Let

$$
B_{1} \stackrel{i_{1}}{\rightarrow} M_{1} \stackrel{i_{2}}{\rightarrow} X
$$

be inclusion maps and $\partial_{r}: \Pi_{r}\left(X, M_{1}\right) \rightarrow \Pi_{r-1}\left(M_{1}\right)$ be the boundary homomorphism. Then we have the following:

(a) $i_{2 *}$ is an epimorphism for $2 \leqq r<\min \left(\Gamma_{1}+1, \Gamma_{3}, \Gamma_{5}\right)$,

(b) $i_{2 *}$ is an isomorphism for $2 \leqq r<\min \left(\Gamma_{1}, \Gamma_{3}, \Gamma_{5}\right)$,

(c) $\gamma_{r, 1} j_{r}: \Pi_{r}\left(M_{1}\right) \rightarrow \Pi_{r}(X, N)$ is an epimorphism if $2 \leqq r<\min \left(\Gamma_{1}+1, \Gamma_{3}\right)$, 
(d) there exists an exact sequence

$$
0 \rightarrow \operatorname{Ker} i_{2 *} \rightarrow \Pi_{r}\left(M_{1}\right) \rightarrow \Pi_{r}(X) \rightarrow \operatorname{Ker} \partial_{r} \rightarrow 0,
$$

(e) $\operatorname{Im} i_{1 *} \subset \operatorname{Ker} i_{2 *}$ if $r<\Gamma_{5}$,

(f) $\operatorname{Im} i_{1 *} \subset \operatorname{Ker} \gamma_{r, 1} j_{r}$.

Proof. Consider the following commutative diagram which is analogous to Diagram 1 with the addition of the exact sequence of the pair $\left(X, M_{1}\right)$ in the second column and the extension of the third column by a triad group.

$$
\begin{aligned}
& \rightarrow \Pi_{r}\left(B_{1}\right) \stackrel{i_{1}^{*}}{\rightarrow} \Pi_{r}\left(M_{1}\right) \stackrel{i_{r}}{\rightarrow} \Pi_{r}\left(M_{1}, B_{1}\right) \rightarrow \Pi_{r-1}\left(B_{1}\right) \rightarrow
\end{aligned}
$$

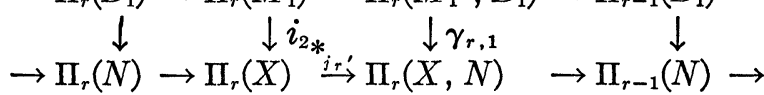

$$
\begin{aligned}
& \downarrow j_{r *} \quad \downarrow j_{r, 1} \\
& \Pi_{r}\left(X, M_{1}\right) \Pi_{r}\left(X ; M_{1}, N\right) \\
& \downarrow \partial_{r} \\
& \Pi_{r-1}\left(M_{1}\right)
\end{aligned}
$$

Diagram 2.

For part (a) note that $j_{r}^{\prime}$ is an isomorphism if $r<\Gamma_{5}, j_{r}$ is an epimorphism if $r<\Gamma_{1}+1$, and, from Lemma 2.1, $\gamma_{r, 1}$ is an epimorphism if $2 \leqq$ $r<\min \left(\Gamma_{1}+1, \Gamma_{b}\right)$. The result now follows from the fact that

$$
i_{2 *}=\left(j_{r}^{\prime}\right)^{-1} \gamma_{r, 1} j_{r}
$$

For part (b) note that $j_{r}$ is an isomorphism if $r<\Gamma_{1}$ and, from Lemma 2.3, $\gamma_{r, 1}$ is an isomorphism for $2 \leqq r<\min \left(\Gamma_{1}, \Gamma_{3}\right)$. The result follows now as in part (a).

For part (c) note $j_{r}$ is an epimorphism for $r<\Gamma_{1}+1$ and $\gamma_{r, 1}$ is an epimorphism if $2 \leqq r<\min \left(\Gamma_{1}+1, \Gamma_{3}\right)$.

For part (d) note that the exact sequence of the pair $\left(X, M_{1}\right)$ implies that

$$
\text { Coker } i_{2_{*}} \simeq \Pi_{r}(X) / \operatorname{Im} i_{2_{*}} \simeq \Pi_{r}(X) / \operatorname{Ker} j_{r_{*}} \simeq \operatorname{Ker} \partial_{r} \text {. }
$$

For part (e) note that

$$
j_{r}^{\prime} i_{2 *} i_{1 *}=\gamma_{r, 1} j_{r} i_{1 *}=0,
$$

and, if $r<\Gamma_{5}$, then $j_{r}^{\prime}$ is an isomorphism. Hence $i_{2_{*}} i_{1_{*}}=0$. For part (f) we have that

$$
\operatorname{Im} i_{1 *} \subset \operatorname{Ker} j_{r} \subset \operatorname{Ker} \gamma_{r, 1} j_{r} .
$$

Corollary. If $2 \leqq r<\min \left(\Gamma_{1}+1, \Gamma_{3}, \Gamma_{5}\right)$ then $\partial_{r}$ is a monomorphism.

Proof. Part (a) and (d) of Theorem 2.2 gives the results immediately. We now combine the above two theorems to obtain the following result.

Theorem 2.3. Let $M_{2}=M_{1} \Delta N$. If $2 \leqq r<\min \left(\Gamma_{2}, \Gamma_{4}, \Gamma_{5}\right)$ then there exists 
a map $L: \Pi_{r}\left(M_{1}\right) \rightarrow \Pi_{r}\left(M_{2}\right)$ with the property that $\operatorname{Im} i_{1_{*}} \subset$ Ker $L$ and Coker $L \simeq \operatorname{Ker} \partial_{r}$. If $2 \leqq r<\min \left(\Gamma_{1}+1, \Gamma_{2}, \Gamma_{3}, \Gamma_{4}, \Gamma_{5}\right)$ then $L$ is an epimorphism and if $2 \leqq r<\min \left(\Gamma_{1}, \Gamma_{2}, \Gamma_{3}, \Gamma_{4}, \Gamma_{5}\right)$ then $L$ is an isomorphism. If $2 \leqq r<$ min $\left(\Gamma_{1}+1, \Gamma_{2}, \Gamma_{3}\right)$ then $\Pi_{r}\left(M_{2}\right)$ is isomorphic to a subgroup of $\Pi_{r}\left(M_{1}\right) / K$ where $K$ is a subgroup of $\Pi_{r}\left(M_{1}\right)$ containing $\operatorname{Im} i_{1_{*}}$.

Proof. Consider the following diagram:

$$
\Pi_{r}\left(M_{1}\right) \stackrel{i_{3}^{*}}{\rightarrow} \Pi_{r}(X) \stackrel{i *}{\leftarrow} \Pi_{r}\left(M_{2}\right) .
$$

By Theorem 2.1 $i_{*}$ is an isomorphism if $2 \leqq r<\min \left(\Gamma_{2}, \Gamma_{4}, \Gamma_{5}\right)$. Define $L=i_{*}^{-1} i_{2 *}$. The result follows directly from Theorem 2.2. The last part of Theorem 2.3 follows from the last part of Theorem 2.1 and Theorem 2.2 parts (c) and (f).

Definition 2.1. The map $L$ of Theorem 2.3 is called the natural homomorphism.

3. $\Phi-M o r p h i s m s$ and Stability. We now assume that for each $r$ there is given a group $G_{r}$ and group homomorphisms $\Phi_{i}: \Pi_{r}\left(M_{i}\right) \rightarrow G_{r}, i=1$, 2, with the property that there exists for each $r$ a group homomorphism $\Phi$ forming the following commutative diagram where $i_{1}$ and $i_{2}$ are induced by inclusion.

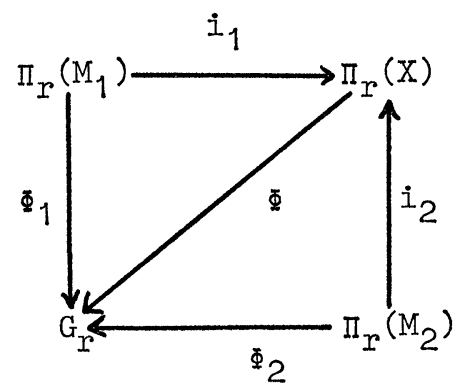

Diagram 3.

Definition 3.1. A group homomorphism $h: \Pi_{r}\left(M_{1}\right) \rightarrow \Pi_{r}\left(M_{2}\right)$ is said to be a $\Phi$-morphism if the following diagram is commutative

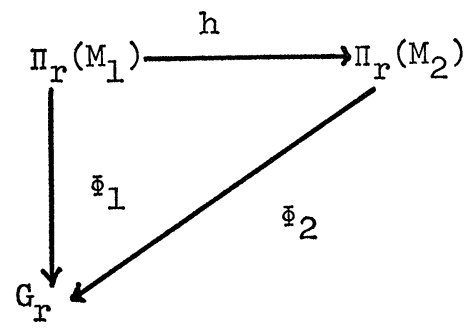

One way in which $G_{r}, \Phi_{1}, \Phi_{2}, \Phi$ and $\Phi$-morphisms arise is as follows. Suppose there is given a map $f: X \rightarrow Y$ where $Y$ is some arcwise connected space. Then 


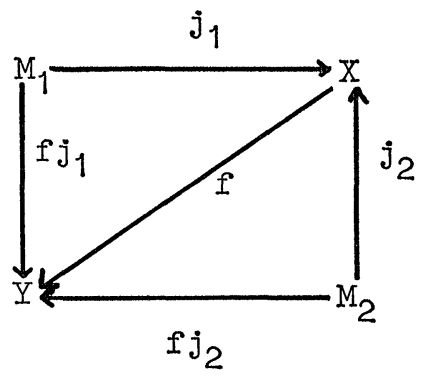

Diagram 4.

we have the following commutative diagram where $j_{1}$ and $j_{2}$ are inclusions. Applying $\Pi_{r}$ we may define $G_{r}=\Pi_{r}(Y), \Phi_{1}=\left(f j_{1}\right)_{*}, \Phi_{2}=\left(f j_{2}\right)_{*}$ and $\Phi=f_{*}$. In this case, if $g$ is a map such that $j_{2} g=j_{1}$, then $g_{*}$ is a $f_{*}$-morphism since

$$
\Phi_{2} g_{*}=\left(f j_{2} g\right)_{*}=\left(f j_{1}\right)_{*}=\Phi_{1} .
$$

In particular if $Y$ is some homotopically trivial space (e.g. some euclidean space) then any group homomorphism $h$ is a $\Phi$-morphism since $G_{r}=0$. In $\S 9$ we make use of the above construction where $Y$ is a certain Grassmann manifold.

Definition 3.2. The $r$ th homotopy group $\Pi_{r}$ of $M_{1}$ is said to be $\Phi$-stable if there exists a $\Phi$-morphism $h$ which is an isomorphism.

Definition 3.3. The group $\Pi_{r}$ is said to be stable if it is $\Phi$-stable and $L$, the natural homomorphism, may be used for $h$.

Theorem 3.1. Let $M_{2}=M_{1} \Delta N$. The natural homomorphism $L$ is a $\Phi$-morphism with $\operatorname{Ker} L \subset \operatorname{Ker} \Phi_{1}$, and, if $2 \leqq r<\min \left(\Gamma_{i}\right), i=1, \cdots, 5$, then $\Pi_{r}$ is stable.

Proof. We have the following commutative diagram with $j_{1}, j_{2}$ inclusions and $i_{1}, i_{2}$ induced by inclusion.

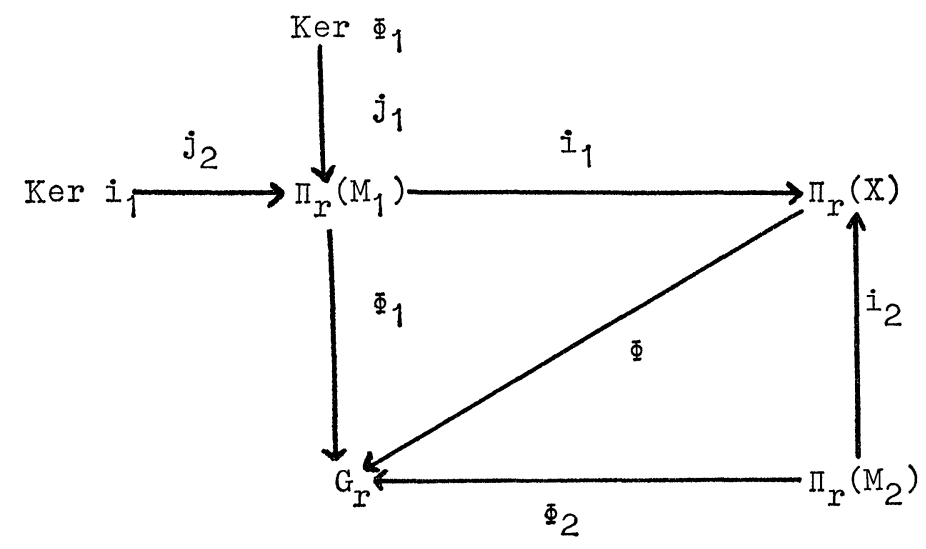


To show $L$ is a $\Phi$-morphism we must show that $\Phi_{2} L=\Phi_{1}$. By definition $L=i_{2}^{-1} i_{1}$. Hence from the diagram we have that

$$
\Phi_{2} L=\Phi_{2} i_{2}^{-1} i_{1}=\Phi i_{1}=\Phi_{1} .
$$

To show $\operatorname{Ker} L \subset \operatorname{Ker} \Phi_{1}$ we need only to show $\Phi_{1} j_{2}=0$ since $\operatorname{Ker} i_{1}=\operatorname{Ker} L$. But $\Phi_{1} j_{2}=\Phi i_{1} j_{2}=0$. The stability follows directly from Theorem 2.3 and the fact that $K$ is a $\Phi$-morphism.

Remark. It should be noted that all of the above results depend only on dimension conditions and the existance of the maps $\Phi_{1}, \Phi_{2}, \Phi$. The differentiable structure of the manifolds has not entered directly.

4. Spherical Modifications. We shall now describe a special type of modification and study in more detail the algebraic and geometrical implications of the previous theorems for this particular case.

Denote by $E^{n}$ an $n$-cell and by $\partial$ the operation of taking the boundary. For any integer $p, 0 \leqq p \leqq n$, we have the following decomposition of an $n$-sphere $S^{n}$.

$$
\begin{aligned}
S^{n} & =\partial\left(E^{n+1}\right)=\partial\left(E^{p+1} \times E^{n-p}\right)=\left(\partial\left(E^{p+1}\right) \times E^{n-p}\right) \cup\left(E^{p+1} \times \partial\left(E^{n-p}\right)\right) \\
& =\left(S^{p} \times E^{n-p}\right) \cup\left(E^{p+1} \times S^{n-p-1}\right) .
\end{aligned}
$$

Letting $N=S^{n}, B_{1}=S^{p} \times E^{n-p}$ and $B_{2}=E^{p+1} \times S^{n-p-1}$ we have $N=B_{1} \cup B_{2}$.

Definition 4.1. A $p$-sphere $S^{p}$ imbedded in an $n$-manifold $M_{1}$ is said to be directly imbedded if its normal bundle $B_{1}^{\prime}$ in $M_{1}$ is trivial; that is, if its of the form $S^{p} \times E^{n-p}$.

Given a diffeomorphism $f: B_{1}^{\prime} \rightarrow B_{1}$ which is a fibre bundle morphism, $B_{1}^{\prime}$, $B_{1}$ being considered as bundles over $S^{p}$, we can form the following space $X$. Define on $M_{1} \cup N$, disjoint union, the following equivalence relation $R$. For $x \varepsilon M_{1}, y \varepsilon N$ define $x R y$ if and only if $f(x)=y$. Let $X=M_{1} \cup N / R$. We have natural inclusions of $M_{1}$ and $N$ into $X$ which form the following commutative diagram.

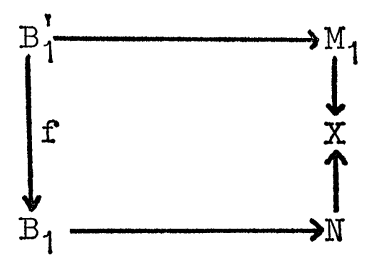

We now identify $M_{1}$ and $N$ with their images in $X$. Thus $M_{1} \cap N=B_{1}$. It is clear that in this case $M_{2}$ can be taken as an $n$-manifold.

Definition 4.2. The manifold $M_{2}$ gotten from $M_{1}$ as described above is said to be obtained from $M_{1}$ by a spherical modification of type $[P, f]$ or $(P, n-P-1)$ if only the dimension is important. 
Remark 4.1. The above definition of spherical modification is equivalent to the notion by the same name as described by Wallace [13, 774-777], although discussed there from a somewhat different point of view.

Before we can apply the results on modifications to spherical modifications it is necessary to define for each $r$ a group $G_{r}$ and group homomorphisms obeying the conditions of $\S 3$. To this end we give a discussion of certain other concepts which will not only allow us to define the groups $G_{r}$ (in $\$ 9$ ) but at the same time will allow us to obtain a factorization of the $\Phi$ 's which will be useful later $(\$ 15)$.

5. Universal Bundles. Let $B$ be an $n+1$-universal bundle for group $G$ $(C f .[10,101])$. Then by Theorem 19.3 of $[10]$ there is a one-to-one correspondence between homotopy classes of maps of a given $n$-complex $K$ into the base space of $B$ and equivalence classes of principal bundles on $K$ with group $G$, the bundle corresponding to a map $f$ is the one induced by $f$ on $K(C f .[10,47])$. We denote by $U_{n+1}$ the base space of an $n+1$-universal bundle for group $R_{n}$ (rotation group) and by $U$ the bundle space. Denote by $U_{n+1}^{\prime}$ the base space of a $n+1$ universal bundle for group $O_{n}$ (orthogonal group) and by $U^{\prime}$ the bundle space.

Definition 5.1. Let $M$ be an orientable $n$-manifold, then a map $T: M \rightarrow U_{n+1}$ such that the induced bundle $B(T)$ on $M$ is equivalent to the associated principal bundle to the tangent bundle of $M$ is called a tangential map of $M$. If $M$ is not orientable replace $U_{n+1}$ by $U_{n+1}^{\prime}$.

Remark 5.1. Let $f: N \rightarrow M$ be an imbedding of an $m$-manifold $N$ in the $n$-manifold $M$. We then have the following commutative diagram.

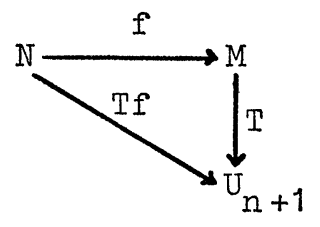

By transitivity of induced bundles we have that $B(T f)$ is equivalent to the bundle $B(f)$ induced by $f$ from the bundle $B(T)$ on $M$. Thus $B(T f)$ may be considered as the principal tangent bundle of $M$ restricted to $f(N)$, assuming $T$ is a tangential map.

6. Characteristic Homomorphisms of a Bundle. Let $B$ be a principal bundle on $X, G_{0}$ the fibre over $x_{0} \varepsilon X$ and $\xi: G \rightarrow G_{0}(G=$ group of bundle) be an admissible map. (Cf. [10,38]). Let $y_{0}=\xi(e), e=$ identity of $G$. Since $\xi$ is a homeomorphism, $\xi_{k}: \Pi_{k}(G, e) \rightarrow \Pi_{k}\left(G_{0}, y_{0}\right)$ is an isomorphism. From the exact sequence of the bundle we can form the following commutative diagram for $k \geqq 1$. $\chi_{k}=\xi_{k-1}^{-1} \Delta_{k}$ by definition and is called the $k$ th characteristic homomorphism of the bundle $B$. If $G$ is arcwise connected then $\chi_{k}$ is uniquely determined by $B$. (Cf. $[10,95])$. 


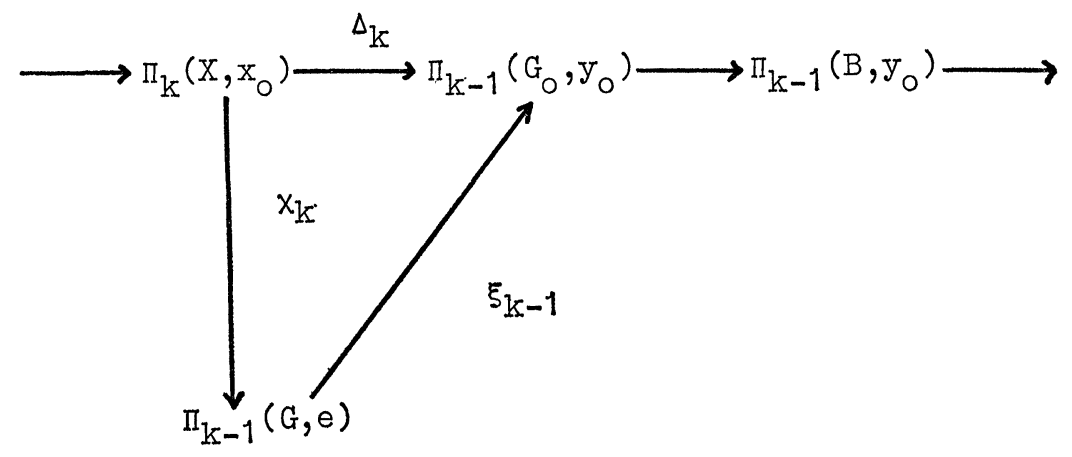

Let $f$ be a map of a manifold $N \rightarrow U_{n+1}$. We then form the following commutative diagram.

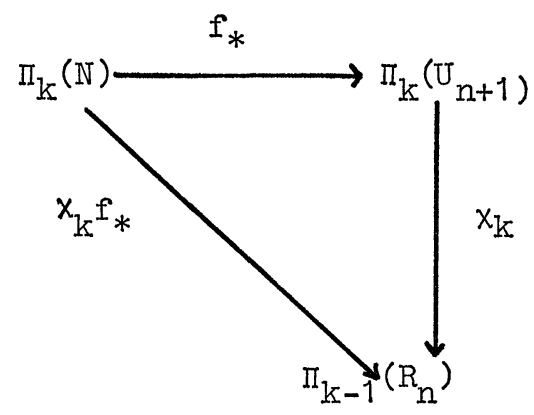

Where $\chi_{k}$ is the $k$ th characteristic homomorphism of the bundle $U \rightarrow U_{n+1}$. Let $\chi_{k}^{\prime}$ be the $k$ th characteristic homomorphism of the bundle $B(f)$.

Theorem 6.1. $\chi_{k}^{\prime}=\chi_{k} f_{*}$.

Proof. Let $\left(G_{i}, y_{i}\right)$ be fibres over $x_{i}, i=1,2$, where $x_{1} \varepsilon N, f\left(x_{1}\right)=x_{2} \varepsilon U_{n+1}$. Let $\xi^{i}$ be an admissable map $\xi^{i}:\left(R_{n}, e\right) \rightarrow\left(G_{i}, y_{i}\right), i=1,2$, and let $f^{\prime}$ be the restriction of the $f$-induced bundle space map $f^{\prime \prime}: B(f) \rightarrow U$ to the fibre $\left(G_{1}, y_{1}\right)$. From the definition of induced bundle we have the following commutative diagram.

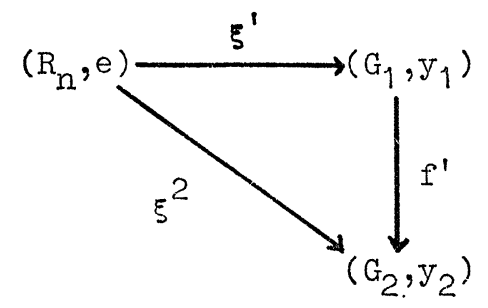

Inserting this diagram in the diagram defining $\chi_{k}$ and $\chi_{k}^{\prime}$ (see first diagram of this section) we have the following diagram where all triangles and the large rectangle are commutative except possibly triangle $I$. 


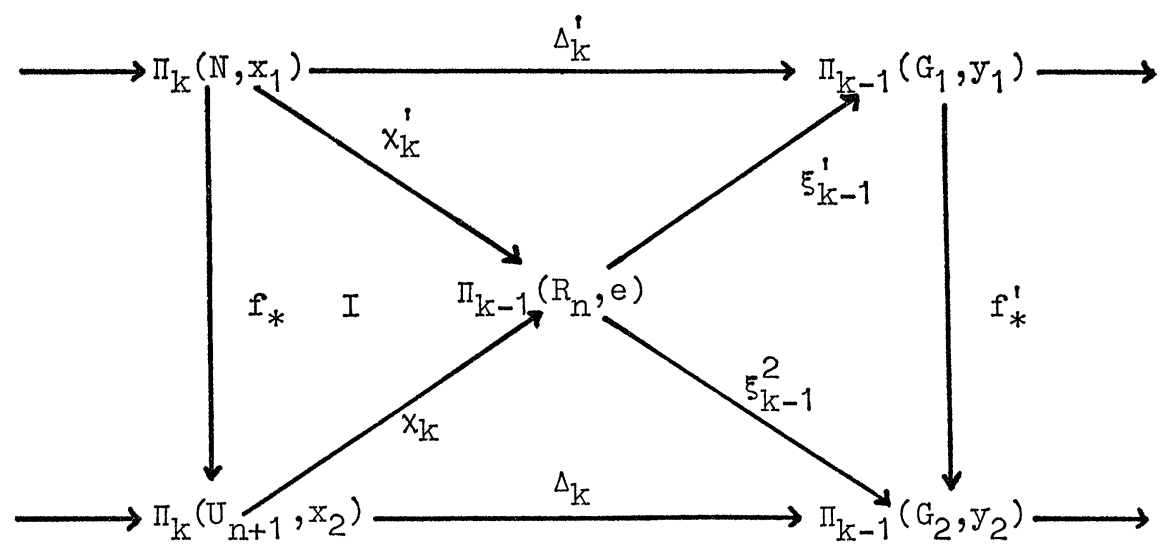

However from the diagram it follows that

$$
\begin{aligned}
\left(\xi_{k-1}^{2}\right)^{-1} \Delta_{k} f_{*} & =\left(\xi_{k-1}^{2}\right)^{-1} f_{*}^{\prime} \Delta_{k}^{\prime} \\
\chi_{k} f_{*} & =\left(\xi_{k-1}^{\prime}\right)^{-1} \Delta_{k}^{\prime} \\
\chi_{k} f_{*} & =\chi_{k}^{\prime}
\end{aligned}
$$

This proves the theorem.

7. Characteristic Class of a Bundle over a Sphere. If the base space of a bundle $B$ is an $r$-sphere then the characteristic class of the bundle is defined to be $\chi_{r}(g) \varepsilon \Pi_{r-1}(G)$ where $g$ is a fixed generator of $\Pi_{r}\left(S^{r}\right)$; that is, an orientation of $S^{r}$ is assumed. The characteristic class is uniquely determined provided the group $G$ of the bundle is arcwise connected. The class can be represented by a map $F: S^{r-1} \rightarrow G$ where $S^{r-1}$ is taken as a great sphere on $S^{r}$ and $F=g_{12} \mid S^{r-1}$ (restriction) where $g_{12}$ is a coordinate transform of the bundle when it is put in normal form; that is, when the coordinate neighborhoods of $S^{r}$ are taken to be two open "hemispheres" $U_{1}$ and $U_{2}$ with $S^{r-1} \subset U_{1} \cap U_{2}$. (Cf. [10, 96-97]).

Suppose $x \varepsilon \Pi_{r}(M)$ and that $x$ can be represented by an imbedding $f: S^{r} \rightarrow M$; that is, $f_{*}(g)=x$ where $g$ is a generator of $\Pi_{r}\left(S^{r}\right)$ and $f_{*}: \Pi_{r}\left(S^{r}\right) \rightarrow \Pi_{r}(M)$.

Let $S^{r}=N$ in Remark 5.1. Applying $\Pi_{r}$ we obtain the following commutative diagram where $T_{*}$ is induced by a tangential map. (See page 693.)

If $\chi_{r}^{\prime}$ is the $r$ th characteristic homomorphism of the bundle $B(T f)$ (induced by $T f$ from $U$ ) over $S^{r}$ then by Theorem 6.1 we have that $\chi_{r}(T f)_{*}(g)=\chi_{r}^{\prime}(g)$. This together with Remark 5.1 gives the following.

Theorem 7.1. Suppose, $x \in \Pi_{r}(M)$ can be represented by a map of $S^{r}$ into $M$ which is an imbedding. Then $\chi_{r} T_{*}(x)$ is the characteristic class of the bundle over $S^{r}$ which is the restriction of the principal tangent bundle of $M$ to $S^{r}$.

Remark. Since we are assuming $S^{r}$ is imbedded we can identify $S^{r}$ with its 


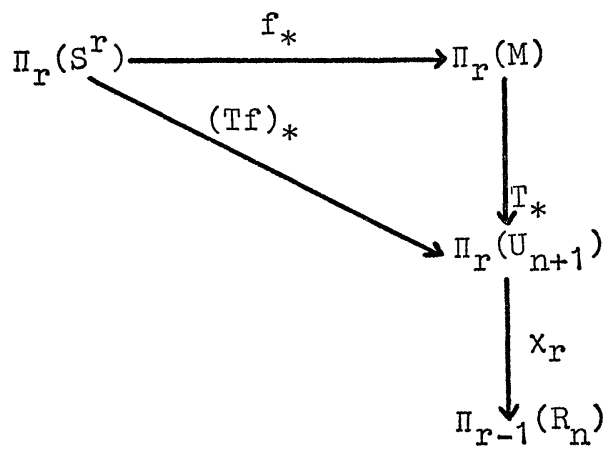

imbedded image. Thus the base space is actually a sphere and characteristic class makes sense.

8. The Whitney Sum of Bundles. Let $B_{r}, B_{k}$ be principal bundles over the same base space $X$ with groups $R_{r}$ and $R_{k}$ respectively. We assume that $B_{r}$ and $B_{k}$ have the same coordinate neighborhoods $\left\{U_{i}\right\}$ and coordinate transforms $g_{i i}, g_{i j}^{\prime}$ respectively. The Whitney sum of $B_{r}$ and $B_{k}$, written $B_{r} \oplus B_{k}$, is the principal bundle over $X$ with group $R_{r+k}$ determined by the coordinate neighborhoods $\left\{U_{i}\right\}$ and coordinate transforms $h_{i j}$ where, for $x \in U_{i} \cap U_{i}$,

$$
h_{i j}(x)=\left[\begin{array}{cc}
g_{i j}(x) & 0 \\
0 & g_{i j}^{\prime}(x)
\end{array}\right] \varepsilon R_{r+k}
$$

(Cf. $[6,51])$.

If $X$ is an $n$-sphere $S^{n}$ then $B_{r}, B_{k}$, and $B_{r}+B_{k}$ have well defined characteristic classes. We now determine the characteristic class of $B_{r} \oplus B_{k}$ in terms of the characteristic classes of $B_{r}$ and $B_{k}$. Let $r^{\prime} \varepsilon \Pi_{n-1}\left(R_{r}\right), k^{\prime} \varepsilon \Pi_{n-1}\left(R_{k}\right)$ be the characteristic classes of $B_{r}$ and $B_{k}$ respectively. Let $i_{1}: R_{r} \rightarrow R_{r+k}$ be the map which associates to each element $y \varepsilon R_{r}$ the element

$$
\left(\begin{array}{ll}
y & 0 \\
0 & I
\end{array}\right) \text {, }
$$

in $R_{r+k}$, where $I$ is the $k$-square identity matrix, and let $i_{2}: R_{k} \rightarrow R_{r+k}$ be the map which associates to each element $y \varepsilon R_{k}$ the element

$$
\left(\begin{array}{ll}
I & 0 \\
0 & y
\end{array}\right)
$$

in $R_{r+k}$, where $I$ is the $r$-square identity matrix. Since $i_{1}$ and $i_{2}$ are just inclusion maps it is obvious that they are continuous group homomorphisms.

Theorem 8.1. If $B_{r}$ and $B_{k}$ are as above (base space $S^{n}$ ) with characteristic classes $r^{\prime}$ and $k^{\prime}$ respectively, then the characteristic class of $B_{r} \oplus B_{k}$, written $r^{\prime} \oplus k^{\prime}$, is given by $i_{1_{*}}\left(r^{\prime}\right)+i_{2 *}\left(k^{\prime}\right)$, where $i_{1_{*}}, i_{2 *}$ are induced by $i_{1}, i_{2}$ under $\Pi_{n-1}$. 
Proof. We can write $r^{\prime}=r_{*}(g)$ and $k^{\prime}=k_{*}(g)$ where $g$ is a fixed generator of $\Pi_{n-1}\left(S^{n-1}\right)$ and $r=g_{12}\left|S^{n-1}, k=g_{12}^{\prime}\right| S^{n-1}$ where $g_{12}, g_{12}^{\prime}$ are coordinate transforms of $B_{r}$ and $B_{k}$ respectively. Then a representative, $J$, of $r^{\prime}+k^{\prime}$ is given by $J=h_{12} \mid S^{n-1}$ (i.e. $J_{*}(g)=r^{\prime} \oplus k^{\prime}$ ) where, by definition of Whitney sum,

$$
h_{12}(x)=\left[\begin{array}{cc}
g_{12}(x) & 0 \\
0 & g_{12}^{\prime}(x)
\end{array}\right]=\left[\begin{array}{cc}
g_{12}(x) & 0 \\
0 & I
\end{array}\right]\left[\begin{array}{cc}
I & 0 \\
0 & g_{12}^{\prime}(x)
\end{array}\right]
$$

Thus

$$
h_{12}\left|S^{n-1}=i_{1} g_{12}\right| S^{n-1} \cdot i_{2} g_{12}^{\prime} \mid S^{n-1}
$$

or

$$
J=i_{1} r \cdot i_{2} k \text {. }
$$

It is well known that the group operation of the homotopy groups of a topological group is induced by the topological group's own operation in exactly the manner which gives, upon applying $\Pi_{n-1}$ to the above equation, the following: (Cf. [7, 139])

$$
r^{\prime} \oplus k^{\prime}=J_{*}(g)=i_{1_{*}} r_{*}(g)+i_{2 *} k_{*}(g)=i_{1 *}\left(r^{\prime}\right)+i_{2 *}\left(k^{\prime}\right) .
$$

Lemma 8.1. Let $f: N \rightarrow M$ be an imbedding. Then the principal tangent bundle of $M$ restricted to $f(N)$ is the Whitney sum of the principal tangent bundle of $N$ and the principal normal bundle of $N$ in $M$.

Proof. The lemma is proven in $[6,73]$ or $[1,183]$ for the case of tangent bundles and normal bundles. Going over to the associated principal bundles gives the result upon observing that the fibre does not enter into the definition of Whitney sum.

Theorem 8.2. Let $x \varepsilon \Pi_{r}(M)$ and suppose it can be represented by an imbedding $f: S^{r} \rightarrow M$. Then

$$
\chi_{r} T_{*}(x)=i_{1 *}(\tau)+i_{2 *}(\eta),
$$

where $\tau$ is the characteristic class of the principal tangent bundle of $S^{r}$ and $\eta$ is the characteristic class of the principal normal bundle of $S^{r}$ in $M$.

Proof. Let $T_{r}$ be the principal tangent bundle of $S^{r}$ and $N_{r}$ the principal normal bundle of $S^{r}$ in $M$. Then by Theorem $7.1 \chi_{r} T_{*}(x)$ is the characteristic class of the restricted principal bundle of $M$ to $S^{r}$ and by Lemma 8.1 it follows that

$$
\chi_{r} T_{*}(x)=\tau \oplus \eta
$$

By Theorem 8.1 the result follows.

By placing suitable restrictions on $r$ Theorem 8.2 can be carried further. 
We assume $M$ is orientable and thus $R_{n}$ can be taken as the group of the tangent bundle of $M$.

Lemma 8.2. If $r \leqq n-1(n=$ dimension of $M)$ then $i_{1_{*}}(\tau)=0$.

Proof. The principal tangent bundle of $S^{r}$ is given by $R_{r+1} \rightarrow R_{r+1} / R_{r}=S^{r}$ and the characteristic class of this bundle lies in Ker $i_{*}$, where $i_{*}: \Pi_{r-1}\left(R_{r}\right) \rightarrow$ $\Pi_{r-1}\left(R_{r+1}\right)$, is induced by inclusion. $(C f .[10,121])$. Hence $i_{1}$ may be factored as follows for $r+1 \leqq n$.

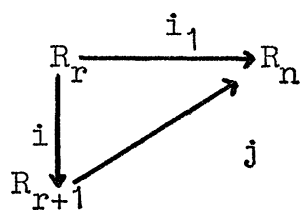

Thus $i_{1 *}(\tau)=j_{*} i_{*}(\tau)=0$.

Lemma 8.3. If $r \leqq n-r-1$ then $i_{2 *}$ is an isomorphism.

Proof. Consider the following exact sequence.

$$
\rightarrow \Pi_{r}\left(R_{n}, R_{n-r}\right) \rightarrow \Pi_{r-1}\left(R_{n-r}\right) \rightarrow \Pi_{r-1}\left(R_{n}\right) \rightarrow \Pi_{r-1}\left(R_{n}, R_{n-r}\right) \rightarrow
$$

By $[10,117]$ we have that $\Pi_{k}\left(R_{n}, R_{n \cdots r}\right)=0$ for $k \leqq n-r-1 \leqq n-1$ and the result follows.

If $x \varepsilon \Pi_{r}(M)$ and $r \leqq n / 2-1$ then $x$ can always be represented by an imbedding. We denote the characteristic class of the principal normal bundle of an imbedded representative by $\eta_{x}$.

Theorem 8.3. Define

for $r \leqq n / 2-1$, then $\Phi_{r}(x)=\eta_{x}$.

$$
\Phi_{r}=i_{2 *}^{-1} \chi_{r} T_{*},
$$

Proof. Theorem 8.2 and Lemma 8.2 imply $\chi_{r} T_{*}(x)=i_{2_{*}}\left(\eta_{x}\right)$ and Lemma 8.3 gives the result.

Corollary. The map $\Phi_{r}$ is the map as defined in $[13,784]$.

Proof. The characteristic class of the normal bundle is the same as the characteristic class of the principal normal bundle. Comparing the definition of $\Phi$ in [13] and the result of Theorem 8.3 we have the corollary.

Lemma 8.4. $\chi_{r}$ is an isomorphism for $1 \leqq r \leqq n$.

Proof. See $[10,104]$.

Lemma 8.5. Let $m>0$ be a given integer. Let $X$ and $Y$ be pathwise connected spaces and $f: X \rightarrow Y$ a continuous map. Let $f_{*}, f_{*}$ be the induced maps

$$
f_{*}: \Pi_{n}(X) \rightarrow \Pi_{n}(Y) \text { and } f_{*}: H_{n}(X) \rightarrow H_{n}(Y) .
$$


If $f_{*}$ is an isomorphism for every $n \leqq m$, then so is $f_{*}$.

Proof. See [7, 167].

9. The $G_{r}$ Groups. As was indicated at the end of $\$ 4$ we can now define groups $G_{r}$ and maps $\Phi_{i}, i=1,2$, (see $\S 3$ ). We have two important cases.

Case 1. Define for each $r, G_{r}=\Pi_{r}\left(U_{n+1}\right)$ and $\Phi_{i}=T_{*}$ where $T$ is a tangential map for $M_{i}, i=1,2$. Let $F: X \rightarrow U_{n+1}$ (see $\$ 2$ for definition of $X$ ) be a map that induces on $X$ the principal bundle which when restricted to $M_{i}$ is the principal tangent bundle of $M_{\imath}, i=1,2$. The commutativity of diagram 3 (\$3) now follows from diagram 4 (\$3) upon noting that by taking $f=F$ and $Y=U_{n+1}$ then the $\Phi_{i}$ as defined in $\$ 3$ directly following diagram 4 and the $\Phi_{i}$ as defined above are the same.

Case 2. For $r \leqq n / 2-1$ let $G_{r}=\Pi_{r-1}\left(R_{n-r}\right)$ and let the $\Phi_{i}$ be the $\Phi_{r}$ of Theorem 8.3 with $M$ replaced by $M_{i}, i=1,2$. To obtain the commutativity of diagram 3 (§3) consider the following commutative diagram where $j_{1}$ and $j_{2}$ denote inclusions.

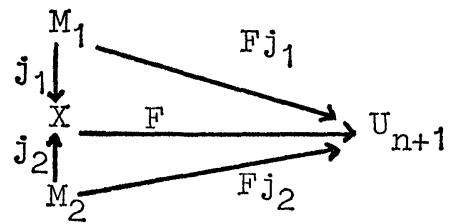

If we apply $\Pi_{r}$ and the results of Theorem 8.3 to the above diagram we have the following commutative diagram which gives diagram 3 ( $\$ 3)$, where $j_{1 *}, j_{2 *}$, and $i_{2 *}^{-1} \chi_{r} F_{*}$ here stand for the maps $i_{1}, i_{2}$ and $\Phi$ respectively of diagram 3. (See top of page 697.)

In sections $10,11,12,13$ the maps $\Phi_{i}$ and the groups $G_{r}$ will be those as defined in Case 2 above.

10. Spherical Modifications on $n$-spheres. Before proceeding to the analysis of performing a $p$-modification on an arbitrary $n$-manifold we shall study in some detail the special case where $M_{1}=S^{n}$. This case is important in that it will give examples of fairly simple $n$-manifolds $M, M^{\prime}$, say, such that there exists for each $r$ an isomorphism $h_{r}: \Pi_{r}(M) \rightarrow \Pi_{r}\left(M^{\prime}\right)$ but that it is impossible to take all the $h_{r}$ 's to be $\Phi$-isomorphisms. Thus the fact that a group remains stable will be seen to be stronger than just being replaced by an isomorphic copy. The results will also be used in $\$ 14$.

Theorem 10.1. Let $M_{2}$ be gotten from $S^{n}$ by a p-modification. Then $M_{2}$ is the bundle space of a fibre bundle over $S^{p+1}$ with fibre $S^{n-p-1}$.

Proof. Let $M_{1}=S_{1}^{n}$ and $N=S^{n}$. Consider the effect of performing a modification of type $[p, f]$. Let

$$
B_{1}=S^{p} \times E^{n-p} \subset S^{n}, \quad B_{1}^{\prime}=S_{1}^{p} \times E_{1}^{n-p} \subset S_{1}^{n} .
$$




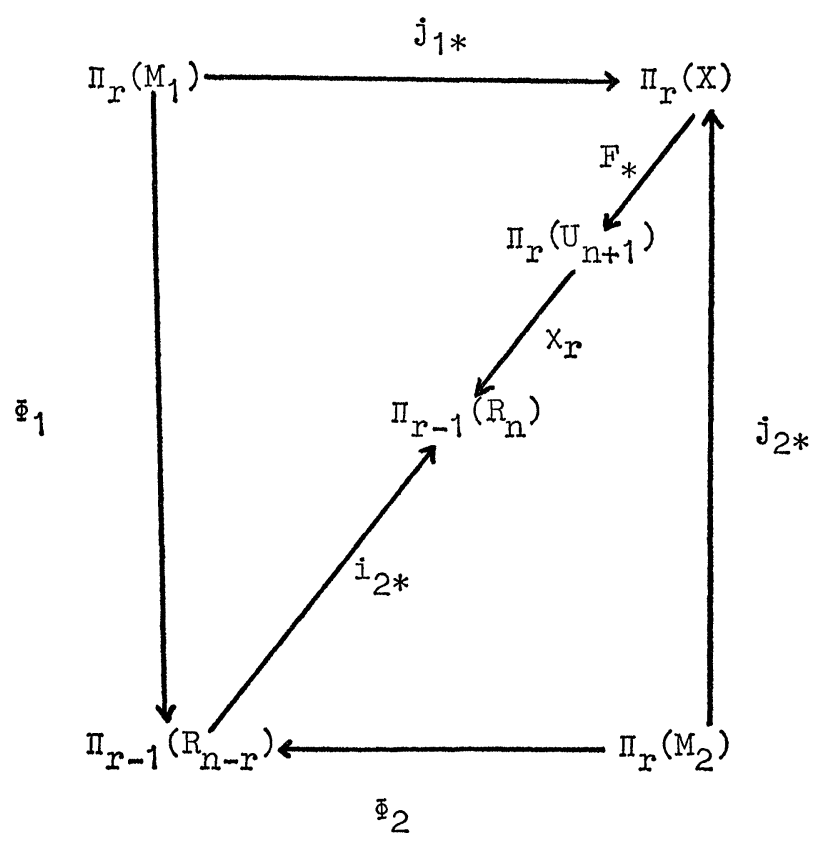

Then $f: B_{1}^{\prime} \rightarrow B_{1}$ is a bundle homeomorphism over $S_{1}^{p}, S^{p}$. Let

$$
B_{2}^{\prime}=E_{1}^{p+1} \times S_{1}^{n-p-1} \subset S_{1}^{n} \text {, and } B_{2}=E^{p+1} \times S^{n-p-1} \subset S^{n} .
$$

Then forming $S_{1}^{n} \Delta S^{n}$ is equivalent to forming the space $M_{2}=B_{2} \cup B_{2}^{\prime} / R$ where $R$ is the equivalence relation on the disjoint union of $B_{2}$ and $B_{2}^{\prime}$ defined as follows: for $x \varepsilon B_{2}^{\prime}, y \varepsilon B_{2}$ then $x R y$ if and only if $f^{\prime}(x)=y$, where $f^{\prime}$ is $f$ restricted to

$$
\partial B_{1}^{\prime}=S_{1}^{p} \times S_{1}^{n-p-1} \subset B_{2}^{\prime} .
$$

To see more clearly what $M_{2}$ is we view $B_{2}^{\prime}$ and $B_{2}$ as trivial bundles over a $(p+1)$-cell $E^{p+1}$. Then taking the boundary of the spaces $B_{2}^{\prime}, B_{2}$ and $E^{p+1}$ is compatible with the projection maps. We thus have the following commutative diagram where the vertical maps are projections.

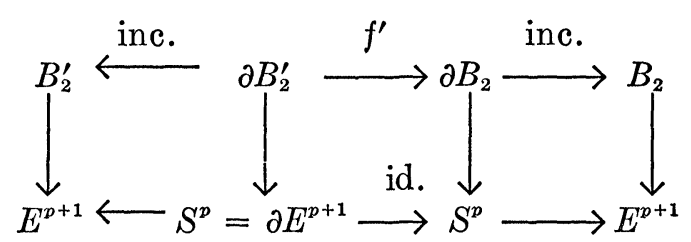

Thus making the identification by $f^{\prime}$ we see that $M_{2}$ is an $n-p-1$-sphere bundle over $S^{p+1}=$ double of $E^{p+1}$ with characteristic class represented by the element in $\Pi_{p}\left(R_{n-p}\right)$ corresponding to the map $f$. This proves the theorem.

The obstruction to extending a cross section from the $g$-skeleton of $S^{p+1}$ to the $(g+1)$-skeleton lies in a cohomology group with coefficients in $\Pi_{\sigma}\left(S^{n-p-1}\right)$ 
which is zero for $g<n-p-1$. If $p<n-p-1$ it follows that $M_{2}$ has a cross section. Hence by $[10,92]$ we have that

$$
\Pi_{k}\left(M_{2}\right) \simeq \Pi_{k}\left(S^{p+1}\right) \oplus \Pi_{k}\left(S^{n-p-1}\right), \quad k \geqq 2 .
$$

If we assume $M_{2}$ is simply connected then the above isomorphism holds for $k \geqq 1$ and $n-p-1>p \geqq 1$.

Example 10.1. Suppose now that $p$ and $n$ are such that $\Pi_{p}\left(R_{n-p}\right)=Z$. Let $g$ be a generator of $Z$ and let $M_{2}, M_{2}^{\prime}$ be manifolds obtained from $S_{1}^{n}$ with characteristic classes $g$ and $2 g$ respectively. Then

$$
\Pi_{p+1}\left(M_{2}\right) \simeq \Pi_{p+1}\left(M_{2}^{\prime}\right) \simeq Z,
$$

(assuming $p+1<n-p-1$ ). However, under the map $\Phi_{2}$, a generator $\gamma$ of $\Pi_{p+1}\left(M_{2}\right)$ is mapped onto $g$ and a generator $\gamma^{\prime}$ of $\Pi_{p+1}\left(M_{2}^{\prime}\right)$ is mapped onto $2 g$. Actually, the $\gamma$ elements are represented by the cross section map of $S^{p+1}$ into $M_{2}, M_{2}^{\prime}$ respectively. Now any isomorphism of $\Pi_{p+1}\left(M_{2}\right)$ onto $\Pi_{p+1}\left(M_{2}^{\prime}\right)$ sends $\gamma \rightarrow \pm \gamma^{\prime}$. Hence no such isomorphism can be a $\Phi$-isomorphism.

Remark 10.1. Some results of $\$ 4$ and Theorem 10.1 can be generalized as follows. Let $A$ and $B$ be compact $r$ and $s$ dimensional manifolds with boundary respectively. If $n=r+s-1$ then $N=\partial(A \times B)$ is a compact $n$-manifold. In the beginning of $\S 4$ we took $A=E^{p+1}$ and $B=E^{n-p}$. We may generalize Theorem 10.1 by studying $N \Delta N$ where $B_{1}=\partial A \times B$ and $B_{2}=A \times \partial B$. If, for example, we take $A=E^{p+1}$ then $B_{1}=S^{p} \times B$ and $N \Delta N$ is the bundle space of a fibre bundle over $S^{p+1}$ with fibre $\partial B$. If $B=E^{n-p}$ we get theorem 10.1 .

11. Homotopy Groups. Let $M_{1}$ be any $n$-manifold and let $S^{p}, p<n-p-1$, be imbedded in $M_{1}$ with the property that it bounds a cell $E^{p+1} \subset M_{1}$. Performing a $p$-modification on $M_{1}$ corresponding to this $S^{p}$ is locally about $S^{p}$ the same as performing a $p$-modification on $M_{1}=S_{1}^{n}$ as above. Hence $M_{2}$ has in it a sphere $S^{p+1}$ composed of $E^{p+1} \subset M_{1}$ and another cell from $N=S^{n}$. $S^{p+1}$ then represents an element $\alpha \varepsilon \Pi_{p+1}\left(M_{2}\right)$. For future use we prove the following theorem.

Theorem 11.1. Let $M_{2}$ be obtained from $M_{1}$ by performing $k$-modifications, $p<n / 2-1$, closing $k(p+1)$-cells in $M_{1}$ and forming $k(p+1)$-spheres in $M_{2}$ representing $k$ elements $\alpha_{1}, \cdots, \alpha_{k}$ in $\Pi_{p+1}\left(M_{2}\right)$. Then $\alpha_{1}, \cdots, \alpha_{k}$ are free and independent elements.

Proof. Since we are dealing with manifolds we may assume that $X$ is a simplicial complex and $N, M_{1}, M_{2}$ are closed subcomplexes of $X$. (See $\S 2$ for definitions.) Then the following Mayer-Vietoris sequences of the triads $\left(X ; M_{1}, N\right)$ and $\left(X ; M_{2}, N\right)$ are exact by Theorem 2.2 of $[4,94]$.

$$
\begin{aligned}
& \rightarrow H_{r}\left(B_{1}\right) \rightarrow H_{r}\left(M_{1}\right) \oplus H_{r}(N) \rightarrow H_{r}(X) \rightarrow H_{r-1}\left(B_{1}\right) \rightarrow \\
& \rightarrow H_{r}\left(B_{2}\right) \rightarrow H_{r}\left(M_{2}\right) \oplus H_{r}(N) \rightarrow H_{r}(X) \rightarrow H_{r-1}\left(B_{2}\right) \rightarrow
\end{aligned}
$$


Assume first that we only make one $p$-modification. Then since $S^{p}$ bounds a $(p+1)$-cell in $M_{1}$ the inclusion induced map $i: H_{p}\left(B_{1}\right) \rightarrow H_{p}\left(M_{1}\right)$ is the zero map. From the above diagram we have, upon observing that $p<(n / 2)-1$ implies $p+1<n-p-1$, that

$$
H_{p+1}\left(M_{2}\right) / H_{p+1}\left(M_{1}\right) \simeq \operatorname{Ker} i=Z \text {, }
$$

hence

$$
H_{p+1}\left(M_{2}\right) \simeq H_{p+1}\left(M_{1}\right) \oplus Z,
$$

and a generator $g$ of $Z$ may be represented by the $(p+1)$-sphere gotten from the closed $(p+1)$-cell. Since the natural homomorphism $\Pi_{p+1}\left(N_{2}\right) \rightarrow H_{p+1}\left(M_{2}\right)$ maps $\alpha_{1}$ onto $g$ we see that $\alpha_{1}$ is free. Now repeating the above argument for $\alpha_{2}, \cdots, \alpha_{k}$ the result follows.

For completeness of what is to follow we state here the theorem on the behavior of the fundamental group of a manifold under a $p$-modification.

Theorem 11.2. Let $M_{2}$ be gotten from $M_{1}$ by a modification of type $(p, n-p-1)$. Then there exists a $\Phi$-homomorphism $L: \Pi_{1}\left(M_{1}\right) \rightarrow \Pi_{1}\left(M_{2}\right)$ which is an isomorphism for $1<p<n-2$ and is epimorphism for $p=1, n>3$ and Ker $L$ is generated by the image of $\Pi_{1}\left(S^{\prime}\right)$ induced by the inclusion of $S^{\prime}$ in $M_{1}, S^{\prime}$ being the 1-sphere determining the 1-modification.

Proof. See [12, 525].

We are now in a position to state the main theorem on the behavior of the homotopy groups of a manifold under a spherical modification.

Theorem 11.3. Let $M_{2}$ be gotten from $M_{1}$ by a spherical modification of type $(p, n-p-1)$. Then the behavior of the various homotopy groups are as follows:

(a) If $r+1 \leqq p \leqq n-r-2$ then the groups $\Pi_{k}$ for $1 \leqq k \leqq r$ are stable.

(b) If $2 \leqq p \leqq r \leqq(n / 2)-1$, then there is a \$-homomorphism $L_{r}: \Pi_{r}\left(M_{1}\right) \rightarrow$ $\Pi_{r}\left(M_{2}\right)$ with the properties that Coker $L=\operatorname{Ker} \partial_{r}, L_{r}$ is an epimorphism if $r=p$, and

$$
i_{1_{*}}\left(\Pi_{r}\left(S^{p}\right)\right) \subset \operatorname{Ker} L_{r} \subset \operatorname{Ker} \Phi_{1},
$$

where $i_{1}: S^{p} \rightarrow M_{1}$ is the imbedding of the p-sphere determining the modification and $\partial_{r}: \Pi_{r}\left(X, M_{1}\right) \rightarrow \Pi_{r-1}\left(M_{1}\right)$ is the boundary homomorphism for the pair $\left(X, M_{1}\right)$ where $X$ is as constructed following Definition 4.1.

(c) If $n-p-1 \leqq r \leqq(n / 2)-1<(n / 2) \leqq p<n-2$ then there is $a$ $\Phi$-homomorphism $L_{r}: \Pi_{r}\left(M_{2}\right) \rightarrow \Pi_{r}\left(M_{1}\right)$ with $\operatorname{Ker} L_{r} \subset \operatorname{Ker} \Phi_{2}$.

Proof. Recall that for a $p$-modification the $\Gamma$ 's occurring in the theorems on modifications are given by $\Gamma_{1}=p, \Gamma_{2}=n-p-1, \Gamma_{3}=\Gamma_{4}=\Gamma_{5}=n$. To show (a) we first assume $2 \leqq p<n / 2$, which means $p \leqq n-p-1$. By Theorem 3.1 the groups $\Pi_{k}$ are stable for $2 \leqq k<\min \left(\Gamma_{i}\right), i=1,2,3,4,5$. Under the conditions on $p$ we have that the $\Pi_{k}$ are stable for $2 \leqq k<p$ or for 
$2 \leqq k \leqq r$, where $r+1 \leqq p$. Assume now that $n / 2 \leqq p \leqq n-r-2$. Then we may view $M_{1}$ as gotten from $M_{2}$ by an $(n-p-1, p)$ modification. But, from the conditions on $p$, it follows that $n-p-1$ obeys the inequality $r+1 \leqq n-p-1<n / 2$. Thus by again applying Theorem 3.1 the groups of $M_{2}$ are stable in the range $2 \leqq k \leqq r$. From Theorem 11.2 we have in fact stability for $k=1$ also. This proves (a).

To show (b) first note that $p \leqq r \leqq(n / 2)-1$ implies that $r<n-p-1$. By Theorem 2.3 there exists a map $L_{r}$, if $2 \leqq r<\min \left(\Gamma_{2}, \Gamma_{4}, \Gamma_{5}\right)=n-p-1$, which is in the range of the given $r$. Also, from Theorem 2.3, we have that $\operatorname{Im} i_{1 *} \subset \operatorname{Ker} L_{r}$, where $i_{1 *}: \Pi_{r}\left(B_{1}\right) \rightarrow \Pi_{r}\left(M_{1}\right)$. But for a spherical modification $B_{1}=S^{p} \times E^{n-p}$, hence $S^{p}$ is a deformation retract of $B_{1}$. Thus $\operatorname{Im} i_{1_{*}}=i_{1_{*}}\left(\Pi_{r}\left(S^{p}\right)\right)$. Further, $L_{r}$ is an epimorphism if

$$
2 \leqq r<\min (p+1, n-p-1, n) .
$$

Since $p \leqq r<n-p-1$ it follows that $L_{r}$ is an epimorphism if $p \leqq r<p+1$ or if $p=r$. Still, from Theorem 2.3, we have that Coker $L_{r}=\operatorname{Ker} \partial_{r}$. From Theorem 3.1 we have that $\operatorname{Ker} L_{r} \subset \operatorname{Ker} \Phi_{1}$. This proves (b).

To show (c) we note that $M_{1}$ may be viewed as being obtained from $M_{2}$ by an $(n-p-1, \mathrm{p})$ modification. Under the conditions on $p$ and $r$ we have that $2 \leqq n-p-1 \leqq r \leqq(n / 2)-1$. Now proceed as in the proof of $(\mathrm{b})$. This proves the theorem.

Note that $i_{1_{*}}\left(\Pi_{r}\left(S^{p}\right)\right) \subset \operatorname{Ker} L_{r}$ in part (b) above may be interpreted geometrically as saying that all of the $r$-spheres carried by the $p$-sphere $S^{p}$ are killed under the modification. More generally, the spheres that are killed are those in $\operatorname{Ker} L_{r}=\operatorname{Ker} i_{2 *}, i_{2 *}: \Pi_{r}\left(M_{1}\right) \rightarrow \Pi_{r}(X)$; that is, a sphere is killed if it can be shrunk to a point after the $n$-sphere $S^{n}=N$ has been attached to $M_{1}$ along $B_{1}=S^{p} \times E^{n-p}$. The fact that Ker $L_{r} \subset \operatorname{Ker} \Phi_{1}$ says that the only $r$-spheres, $r \leqq n / 2-1$, which can be killed by a $p$-modification, $p<n / 2$, are directly imbedded. We thus have the following:

Corollary 1. If a non-directly imbedded $r$-sphere $1 \leqq r \leqq n / 2-1$ can be killed then it must be by a modification of the form $(p, n-p-1)$ with $p \geqq n-r-1 \geqq n / 2$.

If $p=r$ then $L_{r}$ being an epimorphism means that in fact the modification has simplified the group $\Pi_{r}$; that is,

$$
\Pi_{r}\left(M_{2}\right) \simeq \Pi_{r}\left(M_{1}\right) / \operatorname{Ker} L_{r}
$$

However, if $p<r$, then the group $\Pi_{r}$ is first of all simplified by $\operatorname{Ker} L_{r}$, but secondly, since $L_{r}$ is not in general an epimorphism, there may be new elements introduced in $\Pi_{r}\left(M_{2}\right)$, both directly and non-directly imbedded. However no new elements will be introduced into $\Pi_{r}$ if and only if $L_{r}$ is epi; that is, $\delta_{r}$ is a mono. Interpreting this geometrically we have the following:

Corollary 2. No new elements will be introduced into the r-th homotopy group, 
$r \leqq(n / 2)-1$, by a p-modification, $p<(n / 2)$, if and only if no $r$-cell in $M_{1}$ lies on an r-sphere in $M_{1}$ with $S^{n}$ attached (i.e. in $X$ ) which cannot be shrunk to a point in $X$.

Part (c) of Theorem 11.3 says that $\Pi_{r}\left(M_{2}\right)$ may have new elements introduced (i.e. $L_{r}$ may not be a monomorphism) but noting that $\operatorname{Ker} L_{r} \subset \operatorname{Ker} \Phi_{2}$ we have the following:

Corollary 3. If a p-modification, $p \geqq(n / 2)$, is performed on $M_{1}$, then the $r$-th homotopy group, $r \leqq(n / 2)-1$, may have new elements introduced but they all can be represented by directly imbedded spheres in $M_{2}$.

12. Killing Homotopy Groups. Until further mention we assume all manifolds to be compact orientable differentiable manifolds.

Theorem 12.1. Let $M_{1}$ be an n-manifold. Then by a finite sequence of spherical modifications of type $(p, n-p-1)$ for $1 \leqq p<(n / 2)$ one can obtain a manifold $M_{2}$ such that

$$
\Phi_{2}: \Pi_{r}\left(M_{2}\right) \rightarrow \Pi_{r-1}\left(R_{n-r}\right),
$$

is a monomorphism for $1 \leqq r \leqq(n / 2)-1$.

Proof. By the conditions on $M_{1}$ we know that $\operatorname{Ker} \Phi_{1}, \Phi_{1}: \Pi_{r}\left(M_{1}\right) \rightarrow$ $\Pi_{r-1}\left(R_{n-} r\right)$, is finitely generated. Then if $n>3, \Pi_{1}$ can be made zero by Theorem 11.2 and if $n=3$ apply Theorem 6 of [12]. Once $\Pi_{1}=0$, for $n>2$, proceed by killing Ker $\Phi_{1}$ for $r=2,3, \cdots,(n / 2)-1$. This can be done by Theorem 11.3 (b). By part (a) of the same theorem it follows that $\Pi_{k}$ is stable for $p>k$. This completes the proof.

Definition 12.1. A manifold with the property that $\Phi$ is a monomorphism for $r \leqq(n / 2)-1$ will be said to be in general standard homotopy form.

We next consider the possibility of totally killing the $r$-th homotopy group of a manifold. To this end we introduce the following definitions.

Definition 12.2. An $r$-sphere $S^{r}$ is said to be codirectly imbedded in an $n$ manifold $M_{1}$ if it is imbedded and there exists a fibre $E_{0}^{n-r}$ of its normal bundle neighborhood which can be extended to a directly imbedded sphere $S_{0}^{n-r}$; that is, $S_{0}^{n-r} \cap B_{1}=E_{0}^{n-r}$, where $B_{1}$ is a tubular neighborhood of $S^{r}$ which is a fibre bundle over $S^{r}$ of structure type $S^{r} \times E^{n-r}$.

Definition 12.3. An $r$-sphere $S^{r}, r \leqq(n / 2)-1$, is said to be $\Phi$-codirectly imbedded in $M_{1}$ if first of all it is codirectly imbedded and secondly there exists a set of generators $g_{1}, \cdots, g_{1}$ of Ker $\Phi_{1}$ which have imbedded representatives disjoint from $S_{0}^{n-r}$.

Definition 12.4. An element $\alpha \varepsilon \Pi_{r}\left(M_{1}\right)$ is said to be a $\Phi$-codirectly imbedded element if it has an imbedded representative which is $\Phi$-codirectly imbedded in $M_{1}$. 
Theorem 12.2. If $\Pi_{r}\left(M_{1}\right)$ contains a $\Phi$-codirectly imbedded element $\alpha$ which is not directly imbedded, then by means of a finite sequence of modifications of type $(r, n-r-1)$ and one of type $(n-r, r-1)$ one can obtain a manifold $M_{2}$ such that $\Pi_{r}\left(M_{2}\right)=0$ and $\Pi_{k}$ is stable for $k<r$.

Proof. First kill Ker $\Phi_{1}$ by $r$-modifications determined by the set of generators $g_{1}, \cdots, g_{1}$. Call the manifold so obtained $M_{2}^{\prime}$. Then by the conditions on $\alpha$ its representative $S^{r}$ is still in the manifold $M_{2}^{\prime}$ and so is $S_{0}^{n-r}$ (still directly imbedded). Hence $\beta=1_{r}(\alpha) \varepsilon \Pi_{r}\left(M_{2}^{\prime}\right)$ is $\Phi$-codirectly imbedded and $\beta \notin \operatorname{Ker} \Phi_{2}^{\prime}=0$. Hence we need only prove the theorem in the special case that $\alpha \neq 0$ is $\Phi$-codirectly imbedded in $\Pi_{r}\left(M_{1}\right)$ and $\operatorname{Ker} \Phi_{1}=0$. Let $\alpha$ be represented by $S_{0}^{r}$ with normal bundle $B$ and let $S_{0}^{n-r}$ be the directly imbedded sphere such that $S_{0}^{n-r} \cap B=E_{0}^{n-r}$. If we make an $n-r$-modification determined by $S_{0}^{n-r}$ then we have $B_{1}=S_{0}^{n-r} \times E^{r}, B_{2}=S^{r-1} \times E^{n-r+1}$ and $N=S^{n}=B_{1} \cup B_{2}$. Since $B_{1}$ and $B$ both are contained in $M_{1}$ we have the following

$$
\begin{aligned}
M_{2} & =\left(M_{1}-B_{1}\right) \cup B_{2}=\left(M_{1}-\left(B_{1} \cup B\right)\right) \cup\left(B_{2} \cup\left(B-B_{1} \cap B\right)\right) \\
& =\left(M_{1}-B_{1}^{\prime}\right) \cup B_{2}^{\prime},
\end{aligned}
$$

where $B_{1}^{\prime}=B_{1} \cup B$,

$$
B_{2}^{\prime}=B_{2} \cup\left(B-B_{1} \cap B\right)
$$

and, if we define $N^{\prime}$ to be two copies of $B$ and one copy of $S^{n}$ all identified along the set $B_{1} \cap B$ with the two copies of $B$ also identified along the set $\partial B$, then $M_{2}=M_{1} \Delta N^{\prime}$. It is clear from the definition of $B_{1}^{\prime}$ that $i_{1 *}: \Pi_{r}\left(B_{1}^{\prime}\right) \rightarrow \Pi_{r}\left(M_{1}\right)$ maps the generator represented by $S_{0}^{r}$ onto $\alpha$, and that $\Pi_{r}\left(B_{1}^{\prime}\right) \rightarrow \Pi_{r}\left(N^{\prime}\right)$ is an epimorphism. Hence $\Gamma_{1}=r$ and $\Gamma_{3} \geqq r+1$. Further

$$
\text { [closure }\left(B-\left(B \cap B_{1}\right)\right] \cap B_{2}=\partial E_{0}^{r} \times E^{n-r} \text {, }
$$

where $E_{0}^{r}=S_{0}^{r} \cap B_{1}$. Let $S_{0}^{r-1}=\partial E_{0}^{r}$ and $E_{1}^{r}=$ closure $\left(S_{0}^{r}-E_{0}^{r}\right)$. Then since $S_{0}^{r-1}$ is a deformation retract of $B_{2}$ and $S_{0}^{r-1} C$ closure $\left[B-B \cap B_{1}\right.$ ], which is of the form $E^{n-r} \times E_{1}^{r}$, it follows that $B_{2}^{\prime}$ has homotopy type of a point and hence $\Gamma_{2}=\infty$. Thus $\Gamma_{1}=r, \Gamma_{2}=\infty$ and $\Gamma_{3} \geqq r+1$. From the last part of Theorem 2.3 we have that $\Pi_{k}\left(M_{2}\right)$ is isomorphic to a subgroup of $\Pi_{k}\left(M_{1}\right) / K$ if $2 \leqq k<\min \left(\Gamma_{1}+1, \Gamma_{2}, \Gamma_{3}\right)$ and $K \supset \operatorname{Im} i_{1 *}$. Since $\min \left(\Gamma_{1}+1, \Gamma_{2}, \Gamma_{3}\right) \geqq$ $r+1$ in this case we have that $\Pi_{r}\left(M_{2}\right)$ is isomorphic to a subgroup of $\Pi_{r}\left(M_{1}\right) / K$ and $\alpha$ is contained in $K$. Further $\Pi_{r-1}\left(R_{n-r}\right)$ is either $0, Z_{2}$ or $Z$ (Cf. [3]). Since $\Phi_{1}$ is a monomorphism $\Pi_{r}\left(M_{1}\right)$ is either $0, Z_{2}$ or a subgroup of $Z$. If $\Pi_{r}\left(M_{1}\right)=0$ there is no problem. If $\Pi_{r}\left(M_{1}\right) \simeq Z_{2}$ then since $\alpha \neq 0$ and $\alpha \varepsilon K$ it follows that $\Pi_{r}\left(M_{1}\right) / K=0$, thus $\Pi_{r}\left(M_{2}\right)=0$. If $\Pi_{r}\left(M_{1}\right)$ is a subgroup of $Z$ then $\Pi_{r}\left(M_{1}\right) / K$ is a finite group and thus $\Pi_{r}\left(M_{2}\right)$ is a finite group. But this means that Ker $\Phi_{2}=\Pi_{r}\left(M_{2}\right), \Phi_{2}: \Pi_{r}\left(M_{2}\right) \rightarrow Z$. Therefore $\Pi_{r}\left(M_{2}\right)$ can be killed by $r$-modifications. The stability follows from Theorem 3.1 since $\min \left(\Gamma_{i}\right), i=1, \cdots, 5$, is $r$ in the above modifications. Since we are dealing with orientable manifolds 
we may as well assume $\Pi_{1}\left(M_{1}\right)=0$ and thus the restriction $2 \leqq k$ from Theorem 2.3 may be lifted. This completes the proof of the theorem.

Suppose $\alpha \varepsilon \Pi_{r}\left(M_{1}\right)$ is represented by an $r$-sphere $S^{r}$ which is both directly and codirectly imbedded in $M_{1}$. Then one may ask how the manifold obtained by killing $\alpha$ with an $r$-modification compares with the one obtained by using an $n-r$-modification. The next theorem deals with this question.

Theorem 12.3. If $\alpha \varepsilon \Pi_{r}(M), r \leqq(n / 2)-1$, is both directly and codirectly imbedded in $M$ then killing $\alpha$ by an r-modification or by an $n-r$-modification results in the same manifold.

Proof. Perform the $n-r$-modification $\theta$ killing $\alpha$ with resulting manifold $M_{1}$. Then performing the $r-1$ modification $\Psi$ on $M_{1}$, which is $\theta$ in reverse, we again get $M$. Note that $\Psi$ closes on $r$-cell $\left(E_{1}^{r}\right.$ in the proof of Theorem 12.2) in $M_{1}$ to form $S^{r}$ in $M$ representing $\alpha$. Let $\varphi$ be any $r$-modification on $M$ determined by $S^{r}$ (i.e. $\varphi$ kills $\alpha$ ). Then $\Psi$ and $\varphi$ form a complementary pair of modifications in the sense of $[13,779]$ and by the result on the same page we have that the manifold obtained from $M$ by applying $\varphi$ is $M_{1}$. Hence both $\theta$ and $\varphi$ applied to $M$ give $M_{1}$.

Corollary. If $\alpha \varepsilon \Pi_{r}(M)$ is codirectly imbedded then $\alpha$ is free.

Proof. This follows directly from the above proof and Theorem 11.1.

Definition 12.5. If $\Phi_{1}$ is an isomorphism for $r \leqq(n / 2)-1$ then we will say that $M_{1}$ is in standard homotopy form.

The following theorem is the counterpart to Theorem 12.1 for the standard homotopy form.

Theorem 12.4. If $M_{1}$ is an orientable n-manifold, then it can be put in standard homotopy form by a finite sequence of spherical modifications of type $(p, n-p-1)$ where $1 \leqq p<(n / 2)$.

Proof. We may assume $\Pi_{1}\left(M_{1}\right)=0$ since we are dealing with orientable manifolds and $n>2$. Let $M_{2}$ be gotten from $M_{1}$ by a modification of type $\left[1, g_{1}\right]$ where the 1 -sphere $S^{1}$ determining the modification bounds a 2-cell $E^{2}$ which is closed by the modification to form a 2 -sphere $S^{2}$ in $M_{2}$ with the property that if $S^{2}$ represents $\alpha$ in $\Pi_{2}\left(M_{2}\right)$ then $\Phi(\alpha)=g_{1}$, a generator of $\Pi_{1}\left(R_{n-2}\right)$. (See construction in §4.) Next perform a modification of type $\left[2, g_{2}\right]$ on $M_{2}$ closing a 3-cell $E^{3}$ in $M_{2}$ to form a 3 -sphere $S^{3}$ in $M_{3}$ with the property that if $\beta$ in $\Pi_{3}\left(M_{3}\right)$ is represented by $S^{3}$ then $\Phi(\beta)=g_{2}$ is a generator of $\Pi_{2}\left(R_{n-3}\right)$. Continue this procedure up through the homotopy groups of dimension $((n / 2)-1)$. Next kill Ker $\Phi$ starting at dimension 2 and continue consecutively up through all dimensions $<(n / 2)$. Since the only modifications used in the entire construction are of dimension $<(n / 2)$ it follows from Corollary 1 of Theorem 11.3 that once a sphere is introduced with the characteristic class of its normal bundle corresponding to some generator $g_{i}$, then it remains in all 
manifolds obtained after its introduction. Hence the final manifold obtained by the above construction will be in standard homotopy form.

13. Cobounding Manifolds. Two differentiable $n$-manifolds $M_{1}$ and $M_{2}$ are said to be cobounding if together they form the boundary of a differentiable $n+1$-manifold $V$. If the manifolds are orientable and oriented then the orientation on $V$ should induce the orientation on $M_{1}$ and the opposite orientation on $M_{2}$. In [12] it is shown that two compact manifolds are cobounding if and only if one can be obtained from the other by a finite sequence of spherical modifications. We next consider the relationship between the notion of modification and the notion of cobounding manifolds.

We first define some special manifolds and modifications. Throughout this section we will ignore orientability unless stated otherwise.

Definition 13.1. Let $B_{1}$ be a compact $n$-manifold with boundary. Denote by $C_{1}$ the compact $n$-manifold without boundary defined by $C_{1}=\partial\left(B_{1} \times I\right)$ where $I$ is the unit interval. Identifying $B_{1}$ with $B_{1} \times\{0\}$ we have that $B_{1} \subset C_{1}$.

Definition 13.2. Let $M_{2}=M_{1} \Delta N$ with $M_{1} \cap N=B_{1}$. The obstruction manifold $Q\left(M_{1} \Delta N\right)$ of the modification is defined by $Q\left(M_{1} \Delta N\right)=N \Delta C_{1}$, where $B_{1} \subset N$ and $B_{1} \subset C_{1}$ are identified by the identity map. The appropriateness of this definition is made clear by the following theorem.

Theorem 13.1. Let $M_{2}=M_{1} \Delta N$ then $M_{1}$ and $M_{2}$ cobound if and only if $Q\left(M_{1} \Delta N\right)$ cobounds $S^{n}, n=\operatorname{dim} M_{1}$.

Proof. Let $X$ be as in $\S 2$. We then define two subspaces $T$ and $Q$ of $X \times I$ as follows:

$$
\begin{aligned}
& T=\left[\left(M_{1}-\text { interior } B_{1}\right) \times I\right] \cup\left[M_{1} \times\{1\}\right] \cup\left[M_{2} \times\{0\}\right], \\
& Q=\left[B_{1} \times\{1\}\right] \cup\left[\partial B_{1} \times I\right] \cup\left[B_{2} \times\{0\}\right] .
\end{aligned}
$$

Note that $Q \subset T$ and by the obvious identification we may assume $M_{1} \subset T$ and $M_{2} \subset T$.

First we claim that $Q=Q\left(M_{1} \Delta N\right)$. By definition $Q\left(M_{1} \Delta N\right)=N \Delta C_{1}$. But this means that we intersect $C_{1}$ and $N$ at $B_{1}$ and remove the interior of $B_{1}$ thus leaving $B_{2}$ with $\partial B_{2} \times I$ attached to $\partial B_{2}$ along $\partial B_{2} \times\{0\}$ and $B_{1}$ attached by identifying $\partial B_{1}$ with $\partial B_{2} \times\{1\}$. (Note that $\partial B_{1}=\partial B_{2}$ ) However this is just the definition of $Q$ above.

Suppose now that $Q$ cobounds $S^{n}$. This means that $Q$ is the boundary of some manifold $P$ (Cf. [12]). Attach $P$ to $T$ by identifying $Q \subset T$ with $Q \subset P$ and call the resulting manifold $V$. The boundary of $V$ is obviously made up of the two manifolds $M_{1}$ and $M_{2}$. Thus $M_{1}$ and $M_{2}$ cobound if $Q$ cobounds $S^{n}$.

Conversely assume that $M_{1}$ and $M_{2}$ are cobounding by forming the boundary of a manifold $V$. Attach $V$ to $T$ by identifying $M_{1}$ and $M_{2}$ in $V$ with $M_{1}$ and $M_{2}$ in $T$ respectively and call the resulting manifold $W$. The boundary of $W$ is obviously $Q$. Thus $Q$ cobounds $S^{n}$ if $M_{1}$ cobounds $M_{2}$. This completes the proof of the theorem. 
Remark. The cobordism class of $Q\left(M_{1} \Delta N\right)$ represents an element of the Thom cobordism group (Cf. [11]). This element of the group may be viewed as the obstruction to realizing the modification by a finite sequence of spherical modifications. Theorem 13.1 says that the realization is possible if and only if the obstruction is the neutral element of the Thom group.

We now study $Q\left(M_{1} \Delta N\right)$ in greater detail.

Lemma 13.1. $Q\left(M_{1} \Delta N\right)=N$.

Proof. Let $B_{1}$ be a compact $n$-submanifold with boundary of $N$ ( $n=$ dimension of $N)$. Then there is a neighborhood in $B_{1}$ of $\partial B_{1}$ of the form $\partial B_{1} \times I$. Let $B_{1}^{\prime}$ be a deformation retract of $B_{1}$ such that $\partial B_{1}^{\prime}=\partial B_{1} \times\left\{\frac{1}{2}\right\}$. It is clear that $C_{1} \Delta N$ is the same manifold as $C_{1}^{\prime} \Delta N$. Hence we may assume that $\partial B_{1} \times I \subset N$ such that

$$
B_{1} \cap\left(\partial B_{1} \times I\right)=\partial B_{1} \times\{1\}
$$

that is, so that

$$
N=\left(B_{2} \times\{0\}\right) \cup\left(\partial B_{1} \times I\right) \cup\left(B_{1} \times\{1\}\right) .
$$

But then

$$
Q\left(M_{1} \Delta N\right)=N \Delta C_{1}=\left(B_{2} \times\{0\}\right) \cup\left(\partial B_{1} \times J\right) \cup\left(B_{1} \times\{2\}\right),
$$

where $J=[0,2]$. Clearly $Q\left(M_{1} \Delta N\right)=N$.

Theorem 13.2. $M_{1}$ cobounds $M_{2}$ if and only if $N$ cobounds $S^{n}$.

Proof. This follows directly from Theorem 13.1 and Lemma 13.1.

If $M_{2}$ is gotten from $M_{1}$ by a single spherical modification then $N=S^{n}$. Thus by attaching an $n$-cell to $T$ one obtains an $n+1$-manifold $V$ with boundary $M_{1} \cup M_{2}$.

Definition 13.3. The manifold $V$ is called the trace of the spherical modification. If $M_{2}$ is gotten from $M_{1}$ by a finite sequence $S$ of spherical modifications then the trace of $S$ is the manifold made up of the traces of the individual spherical modifications of $S$.

As was seen above if $M_{2}$ is gotten from $M_{1}$ by a single spherical modification then $N=S^{n}$ but the converse is not true; that is, $N=S^{n}$ does not imply that $M_{2}$ can be gotten from $M_{1}$ by a single spherical modification as the following example shows.

Example 13.1. Let $M_{1}=S^{2}=N$ and let $M_{1} \cap N=B_{1}$ be three disjoint 2-cells. Then $M_{2}$ is a sphere with two handles (assuming the modification is to preserve orientability) but obviously no single spherical modification on $S^{2}$ will give a two handled sphere.

We next consider the relationship between two cobounding manifolds in standard homotopy form. More explicitly we have the following theorem. 
Theorem 13.3. Let $M_{1}$ and $M_{2}$ be orientable n-manifolds which cobound by forming the boundary of $V$. If $M_{1}$ and $M_{2}$ are in standard homotopy form and $V$ is in general standard homotopy form and $n \geqq 5$ then the only spherical modifications needed to go from $M_{1}$ to $M_{2}$ having trace $V$ are of the form

$$
(m-2, m)(m-1, m-1)(m, m-2)
$$

if $n=2 m-1$ and of the form $(m-1, m)$ or $(m, m-1)$ if $n=2 m$.

Proof. The proof will be by contradiction. Suppose $M_{2}$ is obtained from $M_{1}$ by a minimal sequence $S$ of increasing modifications with trace $V$; that is, all cancellations have been carried out and all $r$-modifications precede all $r+1$ modifications. This can be done by Theorem 3 of [12]. By Theorem 8 of [12] no modifications of the form $(0, n-1)$ or $(n-1,0)$ occur. Consider now the following commutative diagram for $0<p \leqq(n / 2)-1$.

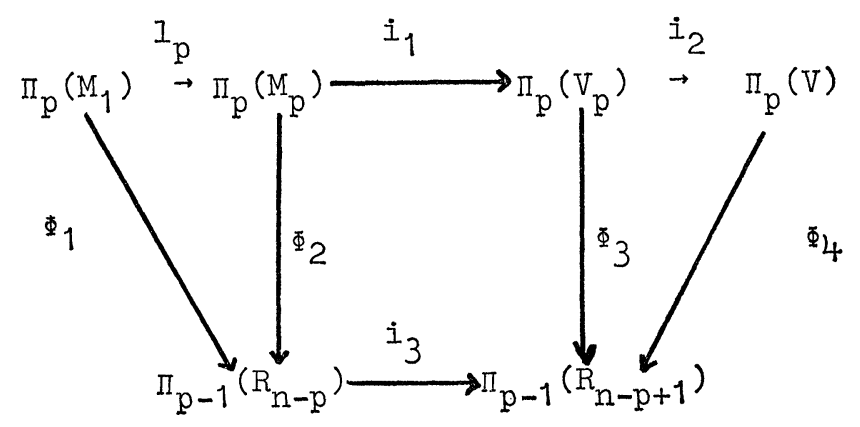

Here $p-1$ is the dimension of the first modifications in $S, L_{p}$ is the composition of the natural homomorphisms corresponding to all $(p-1)$-modifications in $S, M_{p}$ is the manifold gotten from $M_{1}$ by performing all $(p-1)$-modifications in $S, V_{p}$ is the trace of all the $p$-modifications in $S$ and $i_{1}, i_{2}, i_{3}$ are induced by inclusion. The center diagram obviously commutes by definition of $\Phi_{2}, \Phi_{3}$. Let $\Psi$ be any one of the $(p-1)$-modifications of $S$. Then since Ker $\Phi_{1}=0, \Psi$ must close a $p$-cell $E^{p}$ in $M_{1}$ to form a $p$-sphere $S^{p}$ in $M_{p}$ representing an element $\alpha \varepsilon \Pi_{p}\left(M_{p}\right)$. Since $\Phi_{1}$ is an isomorphism

$$
\Pi_{p}\left(M_{p}\right) \simeq \Pi_{p}\left(M_{1}\right) \oplus \operatorname{Ker} \Phi_{2} .
$$

Hence $\alpha \varepsilon \operatorname{Ker} \Phi_{2}$. Thus $\Phi_{4} i_{2} i_{1}(\alpha)=i_{3} \Phi_{2}(\alpha)=0$ and since $\Phi_{4}$ is a monomorphism $\alpha \varepsilon \operatorname{Ker} i_{2} i_{1}$ and by Lemma 8.1 [13], Ker $i_{2} i_{1}=$ Ker $i_{1}$. Hence, by theorem 11.1 , the subgroup $\{\alpha\}$ generated by $\alpha$ is a free subgroup of Ker $i_{1}$. Hence by Lemma 7.2, Lemma 8.3 and Theorem 5.1 of [13] the $p$-modifications of $S$ can be arranged so that one of them will cancel the $(p-1)$-modification $\Psi$ leaving the trace unchanged. But this contradicts the assumption on $S$ unless the first modifications to appear are of type $p$ where $p=m-1$ if $n=2 m$ and $p=m-2$ if $n=2 m-1$. (In order to apply Theorem 11.1 we need $p<(n / 2)-1$.) Repeating the above argument under the assumption $M_{1}$ is gotten from $M_{2}$ the result follows. 
Remark. A result similar to Theorem 12.4 is proven in [13] by means of basic complexes $(C f .[13,800])$ and Theorem 13.3 is essentially proven in [13] (there is an error in the case $n=2 m-1$ ) by means of basic complexes but with somewhat stronger hypothesis; namely, the trace containing a basic complex implies that $\Phi_{1}$ is an isomorphism while all that is used above is that $\Phi_{1}$ is a monomorphism. In $\$ 15$ we show that Theorem 13.3 can be put in terms of homology groups of $M_{1}$ and $M_{2}$. The result will also give information about the basic complex. To do this, we make use of the $G_{r}$ and $\Phi_{i}, i=1,2$, as defined in case $1 \S 9$. However before we do this we combine some results of $\$ 4$ with the concept of cobounding manifolds which leads to a special case of a more general concept; namely, the modification number of a manifold.

14. Modification Numbers. Let $M$ be a compact $n$-manifold. By removing the interiors of two disjoint imbedded $n$-cells $E^{n} \subset M$ one obtains a manifold $V$ with the boundary of $V$ consisting of two copies of $S^{n-1}$. Hence $V$ is the trace of a sequence of spherical modifications leading from $S^{n-1}$ to $S^{n-1}$. Denote by $r$ the minimum number of spherical modifications needed to go from $S^{n-1}$ to $S^{n-1}$ with trace $V$.

Definition 14.1. To each pair of disjoint imbedded $n$-cells in $M$ associate a number $r$ as described above. The modification number $\gamma(M)$ of $M$ is the minimum such $r$ obtained by considering all possible pairs of disjoint imbedded $n$-cells in $M$.

We proceed to study manifolds with small modification numbers. Obviously all modification numbers are non negative. We begin by characterizing a sphere.

Theorem 14.1. Let $n=\operatorname{dim} M$, then $\gamma(M)=0$ if and only if $M=S^{n}$ (homeomorphic).

Proof. Suppose $M=S^{n}$ then removing the interiors of two disjoint imbedded $n$-cells leaves a manifold of the form $S^{n-1} \times I$. Thus no modifications are needed to go from $S^{n-1}$ to $S^{n-1}$ with trace $S^{n-1} \times I$. Conversely if no modifications are used in going from $S^{n-1}$ to $S^{n-1}$ then we may take as trace $S^{n-1} \times I$. Then by adding interiors of two $n$-cells we obviously obtain $M=\partial\left(E^{n} \times I\right)=S^{n}$.

We next study the possibility of having an $n$-manifold $M$ with $\gamma(M)=1$.

Theorem 14.2. If $M$ is an n-manifold with $\gamma(M)=1$ then $n=2^{k}$ for some integer $k \geqq 1$.

Proof. Suppose $\gamma(M)=1$. Then there exist two $n$-cells in $M$ such that after removing their interiors from $M$ one obtains a manifold $V$ which is the trace of a spherical modification leading from $S^{n-1}$ to $S^{n-1}$. From the results of $\$ 10$ we have that any spherical $r$-modification on an $(n-1)$-sphere gives a manifold $M_{2}$ which is the bundle space of a fibre bundle over $S^{r+1}$ with fibre $S^{n-r-2}$. If the modification is to have trace $V$ then $M_{2}=S^{n-1}$. Thus $S^{n-1}$ can be fibred over $S^{r+1}$ with fibre $S^{n-r-2}$. By $[10,219]$ this can only be done if 
$r+1=2^{m}$ and $n-r-2=2^{m}-1$, for some integer $m \geqq 0$. Thus we have that $n=2^{m+1}=2^{k}$, where $k=m+1 \geqq 1$. This proves the theorem.

Actually, we have shown more; namely:

Corollary. If $\gamma(M)=1$ then the single r-modification involved is of the form $r=(n-2) / 2$.

Hence to each $M$ with $\gamma(M)=1$ there corresponds a fibring of an $(n-1)$ sphere over an $(n / 2)$-sphere with fibre an $((n / 2)-1)$-sphere. Obviously any such fibring determines a manifold $M$ with $\gamma(M)=1$ obtained by realizing the fibre bundle by a spherical modification on $S^{n-1}$ and defining $M$ as the trace of the modification with two $n$-cells added at $S^{n-1} \times\{0\}$ and $S^{n-1} \times\{1\}$ respectively. Of course the $n-1$-sphere (total space of the bundle) must have a differentiable structure that can be extended to an $n$-cell.

For $k=1,2,3,4$ the Hopf fibrings

$$
S^{2^{k}-1} \rightarrow S^{2^{k-1}}
$$

give manifolds of dimension $2,4,8$, and 16 with modification number 1 . It is not hard to see that these manifolds are the real, complex, quaternionic, and Cayley projective planes respectively since these manifolds may be realized by adjoining a $2^{k}$-cell to the mapping cylinder of the Hopf fibring.

With one further definition it is possible to state Theorem 12.4 and Theorem 13.3 in terms of homology groups instead of homotopy groups. The result will also give the homology groups of the basic complex.

\section{Standard Homology Form and the Basic Complex.}

Definition 15.1. An orientable $n$-manifold $M_{1}$ is said to be in standard homology form if $T_{*}: H_{r}\left(M_{1}\right) \rightarrow H_{r}\left(U_{n+1}\right)$ is an isomorphism for $r \leqq(n / 2)-1$. ( $T$ is a tangential map.)

Theorem 15.1. If $M_{1}$ is a compact orientable n-manifold, then it can be put in standard homology form by a finite sequence of spherical modifications of type $(p, n-p-1)$, where $1 \leqq p<(n / 2)$.

Proof. Theorem 12.4 says Theorem 15.1 is correct when homology is replaced by homotopy, $H_{r}\left(U_{n+1}\right)$ is replaced by $\Pi_{r-1}\left(R_{n-r}\right)$ and $T_{*}$ is replaced by $\Phi_{r}$. But from Theorem 8.3 and Lemma 8.4 we have that $T_{*}$ is an isomorphism if $\Phi_{r}$ is when $r \leqq(n / 2)-1$. Thus from Lemma 8.5 we have that $T_{*}$ is an isomorphism for $r \leqq(n / 2)-1$.

Theorem 15.2. Let $M_{1}$ and $M_{2}$ be orientable $n$-manifolds, $n \geqq 5$, which cobound by forming the boundary of $V$. If $M_{1}, M_{2}$ and $V$ are in standard homology form, then the only spherical modifications needed to go from $M_{1}$ to $M_{2}$ having trace $V$ are of the form $(m-2, m),(m-1, m-1),(m, m-2)$ if $n=2 m-1$ and of the form $(m-1, m),(m, m-1)$ if $n=2 m$. 
Proof. Consider the following commutative diagram which is similar to the one in the proof of Theorem 13.3.

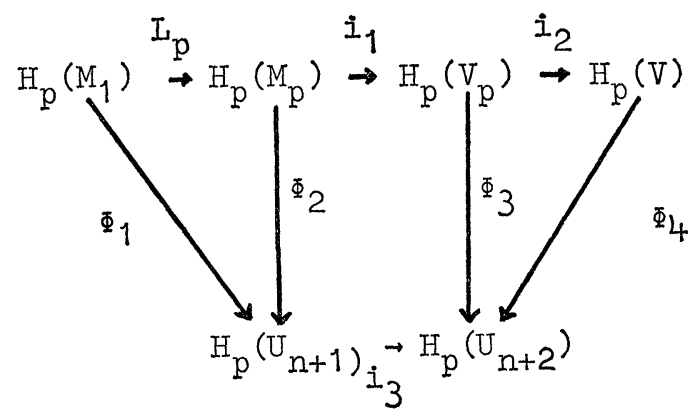

We now proceed as in the proof of Theorem 13.3. Noting that Theorem 11.1 of this paper and Lemma 7.2, Lemma 8.1, Lemma 8.3 and Theorem 5.1 all of [13] hold when homotopy is replaced by homology the result follows as in the case of Theorem 13.3.

Remark. As in the case of Theorem 13.3 we really do not need that the trace $V$ is in standard homology form, but only that $T_{*}$ be a monomorphism, i.e., we only need the trace to be in general standard homology form.

We now apply the above results to a study of the basic complex $L$ introduced by Wallace in $[13,800]$.

Theorem 15.3. Let $\widetilde{M}_{2 n+1, n}$ be the Grassmann manifold of oriented n-planes in $(2 n+1)$-space and let $L$ be a basic complex for an orientable $n$-manifold. Then if $r \leqq(n / 2)-1$,

$$
\Pi_{r}(L) \simeq \Pi_{r}\left(\tilde{M}_{2 n+1, n}\right), \quad \text { and } \quad H_{r}(L) \simeq H_{r}\left(\tilde{M}_{2 n+1, n}\right),
$$

and for $r>(n / 2)-1, H_{r}(L)=0$.

Proof. $L$ can be taken to be an $[(n / 2)-1]$-dimensional complex where [ ] denotes the greatest integer function. Hence $H_{r}(L)=0$, for $r>(n / 2)-1$. Further $L$ has the property that $\Phi_{r} i_{*}$ is an isomorphism for $r \leqq(n / 2)-1$, where $\Phi_{r} i_{*}: \Pi_{r}(L) \rightarrow \Pi_{r}\left(M_{1}\right) \rightarrow \Pi_{r-1}\left(R_{n-r}\right), i_{*}$ induced by inclusion. Hence, as in the proof of Theorem 15.1, $(T i)_{*}$ is an isomorphism and thus $(T i)_{*}: H_{r}(L) \rightarrow H_{r}\left(U_{n+1}\right)$ is an isomorphism for $r \leqq(n / 2)-1$. But in $[10,104]$ it is shown that $\widetilde{M}_{2 n+1, n}$ may be taken for $U_{n+1}$ and the result follows.

16. The Non-orientable Case. The chief difference between the orientable manifold case and the non-orientable manifold case arises from the fact that the group of the tangent bundle to the manifold is the orthogonal group for the non-orientable manifold and not the rotation group. The first, problem one encounters is to construct a map $\Phi_{r}: \Pi_{r}(M) \rightarrow \Pi_{r-1}\left(0_{n-r}\right)$. A map $\Phi_{r}$ may be constructed as in the case of the orientable manifold but instead of using $U_{n+1}$ we substitute $U_{n+1}^{\prime}$ which may be taken to be $M_{2 n+1, n}$ the Grassmann manifold 
of $n$-planes in $(2 n+1)$-space. We can then define $\Phi_{r}$ from the following diagram as $\Phi_{r}=i_{2 *}^{-1} \chi_{r} T_{*}$.

$$
\Pi_{r}(M) \stackrel{T_{*}}{\rightarrow} \Pi_{r}\left(U_{n+1}^{\prime}\right) \stackrel{\chi_{r}}{\rightarrow} \Pi_{r-1}\left(0_{n}\right) \stackrel{i_{2 *}}{\leftarrow} \Pi_{r-1}\left(0_{n-r}\right)
$$

Note that $\Pi_{k}\left(0_{n}\right)=\Pi_{k}\left(R_{n}\right)$, for $k \geqq 1$. Thus $\Phi_{r}$ maps to the same group as the $\Phi_{r}$ for the orientable case, for $r>1$, and $\Phi_{1}: \Pi_{1}(M) \rightarrow \Pi_{0}\left(0_{n}\right)=Z_{2}$.

The geometric interpretation of $\Phi_{r}$ in this case is that $\Phi_{r}(x)$ represents a characteristic class of the normal bundle of an imbedded representative of $x$. The difference is that there is no longer a unique element of $\Pi_{r-1}\left(R_{n-r}\right)$ that represents the characteristic class since the map $\Phi_{r}$ is not uniquely determined. More explicitly, the map $\chi_{r}: \Pi_{r}\left(U_{n+1}^{\prime}\right) \rightarrow \Pi_{r-1}\left(0_{n}\right)$ is only determined to within an equivalence class, equivalence meaning that two $\chi_{r}$ 's are considered equivalent if they differ by the operation of an element of $\Pi_{0}\left(0_{n}\right)$ on $\Pi_{r-1}\left(0_{n}\right)(C f .[10,99])$. In the orientable case $\Pi_{0}\left(R_{n}\right)=0$, thus $\chi_{r}$ is unique and so is $\Phi_{r}$. However in the case that $\Pi_{r-1}\left(0_{n}\right)=0$ or $Z_{2}$ then again $\chi_{r}$ is unique since $\Pi_{0}\left(0_{n}\right)$ must operate trivially.

Once a map $\Phi_{r}$ has been chosen, then most of the results for the orientable case go over for the non-orientable case with only slight change.

If $L$ is a basic complex for a non-orientable manifold (Cf. [9]) and the Grassmann manifold $M_{2 n+1, n}$ is put in the statement of Theorem 15.3 instead of $\widetilde{M}_{2 n+1, n}$ then the result of that theorem still is correct.

\section{REFERENCES}

[1] Auslander \& MacKenzie, Introduction to Differential Manifolds, McGraw-Hill, 1963.

[2] Blakers \& Massey, The homotopy groups of a triad I, Annals of Math., 53 (1951) 161-205.

[3] R. Bотт, The stable homotopy of the classical groups, Annals of Math., 70 (1959) 313-337.

[4] D. Bourgin, Modern Algebraic Topology, Macmillan, 1963.

[5] Eilenberg \& Steenrod, Foundations of Algebraic Topology, Princeton, 1952.

[6] F. Hrrzebruch, Neue topologische methoden in der algebraischen geometrie, Ergebnisse der Mathematik Berlin, 1956.

[7] S. T. Hu, Homotopy Theory, Academic Press, 1959.

[8] R. C. JAmes, Combinatorial topology surfaces, Mathematics Magazine, 29 (1955) 1-39.

[9] J. D. Miller, Minimal sets of modifications, Jour. Math. and Mech., 12 (1963) 751-769.

[10] N. Steenrod, Topology of Fibre Bundles, Princeton, 1951.

[11] R. Tном, Quelques propietes globales des varietes differentiables, Comm. Math. Helv., 28 (1954) 17-86.

[12] A. H. WALLACE, Modifications and cobounding manifolds, Canadian J. Math., 12 (1960) 503-528.

[13] $\longrightarrow$ Modifications and cobounding manifolds II, Jour. Math. and Mech., 10 (1961) $773-809$. 